\title{
Triple canonical surfaces of minimal degree
}

\author{
Margarida Mendes Lopes - Rita Pardini
}

\section{Introduction}

In this paper, continuing the study of pathologies of the canonical map for surfaces of general type that we started in [MP], we study the surfaces with $p_{g} \geq 4$ for which the canonical map is 3-1 onto a surface of minimal degree $n-1$ in $\mathbf{P}^{n}, n \geq 3$, under the assumption that the canonical system contains a smooth curve.

We obtain a complete classification and we construct examples of all the possible numerical cases. Contrarily to what happens for the similar problem for 2-1 canonical map, where there are examples with unbounded invariants (for instance surfaces with $K^{2}=2 p_{g}-4$, see [Ho1], [Pe]), it turns out that these surfaces only exist in a very limited range, namely for $p_{g} \leq 5$ and $K^{2} \leq 9$.

This problem has been studied classically, in a slightly different context, by Pompilj [ $[\mathrm{Pd}]$, for the case of canonical system base point free.

More recently Starnone in his thesis, [St], classified the surfaces with $p_{g} \geq 4$ such that the canonical system has at most simple distinct base points and the general canonical curve is trigonal: it turns out that these surfaces are mapped 3-1 onto a surface of minimal degree in $\mathbf{P}^{p_{g}-1}$ and therefore satisfy our assumptions. Our results, which were obtained at the same time and independently from his, are more general, since we only assume the existence of a smooth canonical curve, and indeed there exist surfaces satisfying our assumptions but not Starnone's, as it is shown by our main result:

Theorem 1.1 a) Let $S$ be a minimal surface of general type such that $p_{g} \geq$ 4, the general canonical curve is smooth, and the canonical map of $S$ is 3-1 onto a surface $\Sigma$ of minimal degree in $\mathbf{P}^{p_{g}-1}$. Then $S$ is regular, $\Sigma$ is a 
cone, $\left|K_{S}\right|$ has at most 2 base points; the invariants of $S$ satisfy

$\begin{array}{ccc} & K_{S}^{2} & p_{g} \\ \left(M_{1}\right) & 9 & 5 \\ \left(M_{2}\right) & 8 & 4 \\ \left(M_{3}\right) & 7 & 4 \\ \left(M_{4}\right) & 6 & 4 \\ (N) & 8 & 4\end{array}$.

Furthermore $S$ contains a rational pencil $|C|$ such that the general curve of $|C|$ is smooth, nonhyperelliptic and such that, in cases $(M), C^{2}=1$, $K_{S} C=3$ and, in case $(N), C^{2}=2, K_{S} C=4$.

In case $(N), K_{S}=2 C$, whilst in case $\left(M_{2}\right)$, which has the same numerical invariants, $K_{S}$ is not 2-divisible in $\operatorname{Pic}(S)$. Furthermore in case $(N)$, $\left|K_{S}\right|$ has two simple base points which are infinitely near.

b) Conversely, any minimal surface $S$ of general type with $p_{g} \geq 4$, containing a rational pencil $|C|$ such that the general curve of $|C|$ is smooth, nonhyperelliptic, with $C^{2}=1, K_{S} C=3$ satisfies the hypothesis in a) and is one the surfaces of type $(M)$. On the other hand if the minimal surface $S$ of general type, with $p_{g} \geq 4$, contains a rational pencil $|C|$ such that the general curve of $|C|$ is smooth, nonhyperelliptic, with $C^{2}=2, K_{S} C=4$, then necessarily $K_{S}^{2}=8, K_{S}=2 C, p_{g}=4$, and the canonical map of $S$ is generically finite of degree 3 or 4 . If the degree is 3 , then $S$ satisfies the hypothesis in a) and is a surface of type $(N)$.

c) All the surfaces described in a) are generically finite triple covers of the cubic cone in $\mathbf{P}^{4}$, and it is possible to construct examples of all such surfaces.

The surfaces of types $\left(M_{1}\right)$ and $\left(M_{4}\right)$ were known to Pompilj, who described them in terms of triple covers of $\mathbf{P}^{2}$, (see $[\mathrm{PO}]$ ). More recently, Horikawa, doing his classification of surfaces with $p_{g}=4, K^{2}=6$, described and proved the existence of surfaces of type $\left(M_{4}\right)$ (see surfaces of type II in [Ho2]), whilst K. Konno constructed surfaces of type $\left(M_{1}\right)$ in [K]. Also Zucconi, in his thesis, [Z], constructs examples of surfaces of types $\left(M_{3}\right)$ and $\left(M_{1}\right)$.

Starnone, in his thesis, working under the assumption that $\left|K_{S}\right|$ has no infinitely near points, finds and constructs all the surfaces of type $(M)$. His results miss the type $(N)$ surfaces exactly because for these surfaces $\left|K_{S}\right|$ always has infinitely near base points. Although both Starnone's constructions for type $(M)$ surfaces and ours are done via triple covers, they differ 
in that Starnone uses exactly the triple covers obtained from the canonical map, whilst we use triple covers of the cubic cone in $\mathbf{P}^{3}$ induced by the linear system $|3 C|$, where $|C|$ is the pencil in thm. 1.1.

We do not know any reference in the literature to surfaces of type $(N)$.

The paper is organized as follows:

In section 2 we show that that every surface $S$ with $3-1$ canonical map satisfying our assumptions contains a pencil of curves as in 1.1 a).

In sections 3 and 4 we study the surfaces having such a pencil and establish various properties of these surfaces. In particular we show that these surfaces are triple covers of the cubic cone in $\mathbf{P}^{4}$.

In section 5 we describe these triple covers precisely, and finally in section 6 we give explicit examples of all these surfaces.

Notation and conventions: All varieties are projective varieties over the complex numbers. The $n$-dimensional projective space is denoted by $\mathbf{P}^{n}$. As usual, $\mathcal{O}_{Y}$ is the structure sheaf of the variety $Y, H^{i}(Y, \mathcal{F})$ is the $i$-th cohomology group of a sheaf $\mathcal{F}$ on $Y$, and $h^{i}(Y, \mathcal{F})$ is the dimension of $H^{i}(Y, \mathcal{F})$; for a line bundle $M$ on $Y$, we denote by $|M|$ the complete linear system $\mathbf{P}\left(H^{0}(Y, M)\right)$. When dealing with smooth varieties, we do not distinguish between line bundles and divisors. If $S$ is a smooth surface, then $K_{S}$ denotes a canonical divisor, $p_{g}(S)=h^{0}\left(S, \mathcal{O}_{S}\left(K_{S}\right)\right)$ is the geometric genus and $q(S)=h^{1}\left(S, \mathcal{O}_{S}\right)$ is the irregularity, $K_{S}^{2}$ is the self-intersection of the canonical divisor. A surface $S$ is said to be regular if $q(S)=0$. A (rational or linear) pencil on a surface $S$ is a linear system on $S$ of projective dimension 1 . The intersection number of two divisors $C, D$ on a smooth surface is denoted simply by $C D$, linear equivalence is denoted by $\equiv$ and numerical equivalence is denoted by $\sim$. We will sometimes refer to an effective non zero divisor on a surface as to a "curve".

Acknowledgements: We wish to thank Patrizia Gianni and Barry Trager, who have shown great patience in teaching us how to do computations with Axiom, and have thus enabled us to construct the examples of section 6 .

The present collaboration takes place in the framework of the HCM contract AGE, no. ERBCHRXCT940557. The first author is a member of CAUL and of the project Praxis 2/2.1/MAT/73/94. The second author is a member of GNSAGA of CNR. This paper was started during a visit, financed by CAUL, of the second author to Lisbon. 


\section{Surfaces whose canonical map is $3-$ to-1 onto a surface of minimal degree}

In this section we prove the following:

Theorem 2.1 Let $S$ be a minimal surface of general type with $p_{g}(S) \geq 4$, let $\phi: S \rightarrow \mathbf{P}^{p_{g}-1}$ be the canonical map of $S$, and let $\Sigma$ be the image of $S$ via $\phi$. Suppose that the general curve of $\left|K_{S}\right|$ is smooth, that $\Sigma$ is a surface, that $\operatorname{deg} \phi$ is equal to 3 and that the degree of $\Sigma$ is equal to $p_{g}-2$, namely it is the lowest possible. Then either $6 \leq K_{S}^{2} \leq 8$ and $p_{g}(S)=4$ or $K_{S}^{2}=9$ and $p_{g}(S)=5$. Furthermore $S$ contains a rational pencil $|C|$ such that the general curve of $|C|$ is irreducible and non-hyperelliptic, and such that either $C^{2}=1, K_{S} C=3$ or $C^{2}=2, K_{S} C=4$.

Remark 2.2 The requirement that the general canonical curve be smooth is equivalent to the fact that $\left|K_{S}\right|$ has no fixed component and that the base points (if any) are simple, possibly infinitely near.

We shall use the following general fact:

Lemma 2.3 Let $X$ be a surface, let $D$ be a curve on $X$ and let $x_{1}, \ldots x_{d} \in$ Sing $D$ be distinct points. Let $p: \tilde{S} \rightarrow S$ be the blow-up at $x_{1}, \ldots x_{d}$ and let $E_{1}, \ldots E_{d}$ be the corresponding exceptional curves. Setting $D^{\prime}=p^{*} D-E_{1}-$ $\cdots-E_{d}$ and $D^{\prime \prime}=p^{*} D-2 E_{1}-\cdots-2 E_{d}$, the following two conditions are equivalent:

(i) $x_{1}, \ldots x_{d}$ do not impose independent conditions on $\left|K_{X}+D\right|$.

(ii) The restriction map $H^{0}\left(D^{\prime}, \mathcal{O}_{D^{\prime}}\right) \rightarrow H^{0}\left(D^{\prime \prime}, \mathcal{O}_{D^{\prime \prime}}\right)$ is not surjective.

Proof: The proof of this lemma is exactly like the proofs of the analogous statements for $d=1$ in [F] and $d=2$ in [M] and so we omit it. $\diamond$

Proof of Theorem 2.1: The proof consists of several steps.

Claim 1: $6 \leq K_{S}^{2} \leq 9$ and $p_{g}=4,5$.

Let $z \in \Sigma$ be a general point, let $\phi^{-1}(z)=\left\{x_{1}, x_{2}, x_{3}\right\}$ and let $C$ be the pullback on $S$ of a general hyperplane section $H$ of $\Sigma$ through $z$. The curve $H$ is smooth rational by the assumption that $\Sigma$ has minimal degree. Therefore, since $C$ is a smooth canonical curve, the linear system $\left|x_{1}+x_{2}+x_{3}\right|$ on $C$ has dimension 1. By Riemann-Roch on $C$, the points $x_{1}, x_{2}, x_{3}$ fail to impose independent conditions on $K_{C}$, and therefore, a fortiori, also on $\left|2 K_{S}\right|$. Let 
$H^{\prime}$ be a tangent hyperplane to $\Sigma$ at $z$ and let $D$ be the pull-back of $H^{\prime}$ to $\Sigma$ : the curve $D$ is singular at $x_{1}, x_{2}, x_{3}$. By lemma 2.3, using the notation introduced there, $h^{0}\left(D^{\prime \prime}, \mathcal{O}_{D^{\prime \prime}}\right) \geq 2$ and thus, in particular $D^{\prime \prime}$ is not $1-$ connected. Arguing exactly as in the proof of thm. 5 of $[\mathrm{B}]$, one shows that there exist curves $A$ and $B$ such that $D=A+B, A B=2$ and at least two of the $x_{i}$ 's belong to $A \cap B$.

Since $z$ can be chosen to be sufficiently general, one can argue as in the proof of thm. 5 of $\mathbb{B}$ and show that $K_{S} A, K_{S} B \geq 2$ and that $K_{S} A=2$ or $K_{S} B=2$ iff $S$ has a pencil of genus 2 . The latter possibility is excluded, since the degree of the canonical map is 3 . So we can assume that $K_{S} A \geq 3$, $K_{S} B \geq 3$.

Say $K_{S} A \leq K_{S} B$. By the index theorem, $A^{2} B^{2}-(A B)^{2} \leq 0$, with equality holding if and only if $B \sim m A$ for some $m \in Q$. Since $K_{S} A=$ $A^{2}+A B=A^{2}+2$ and $K_{S} B=B^{2}+A B=B^{2}+2$ and $K_{S}$ is nef, the numerical possibilities are

$\begin{array}{ccccc} & K_{S}^{2} & K_{S} A & A^{2} & B^{2} \\ \left(M_{1}\right) & 9 & 3 & 1 & 4 \\ \left(M_{2}\right) & 8 & 3 & 1 & 3 \\ \left(M_{3}\right) & 7 & 3 & 1 & 2 \\ \left(M_{4}\right) & 6 & 3 & 1 & 1 \\ (N) & 8 & 4 & 2 & 2\end{array}$.

where in case $\left(M_{1}\right), B \sim 3 A$ and in case $(N) B \sim A$.

So $K_{S}^{2} \leq 9$. Moreover one has

$$
K_{S}^{2} \geq \operatorname{deg} \phi \operatorname{deg} \Sigma=3\left(p_{g}(S)-2\right)
$$

with equality holding if and only if $\left|K_{S}\right|$ is base point free. Since $p_{g}(S) \geq 4$ by assumption, one obtains immediately the assertion about $p_{g}(S)$.

Claim 2: if $p_{g}=5$, then $K_{S}^{2}=9, \Sigma$ is the rational cubic cone and $S$ contains a rational pencil $|C|$ such that the general curve of $|C|$ is irreducible and nonhyperelliptic with $C^{2}=1, K_{S} C=3$.

If $p_{g}=5$, by Claim 1 and 2.1 we have $K_{S}^{2}=9$ and $\left|K_{S}\right|$ base point free. We wish to show that $\Sigma$ is a cone. Assume on the contrary that $\Sigma$ is a rational normal cubic scroll and denote by $C$ the pull-back of a ruling of $\Sigma$ : one has $C^{2}=0$ and $K_{S} C=3$, contradicting the adjunction formula. So $\Sigma$ is the rational normal cubic cone; denote again by $C$ the pull-back of a ruling of $\Sigma$ and consider the pull-back $D$ of a hyperplane section of $\Sigma$ passing through the vertex. One can write $D=3 C+Z$, where $Z$ is an 
effective divisor, possibly empty, that is contracted to the vertex of the cone. Now $9=K_{S}^{2}=K_{S} D=3 K_{S} C+K_{S} Z=9+K_{S} Z$, and so $K_{S} Z=0$ and $K_{S} C=3$. Since $|C|$ is positive dimensional and the general $C$ is irreducible, $C^{2} \geq 0$, and $C^{2} \neq 0$ by parity. So the Hodge index theorem finally yields $C^{2}=1$ and $K_{S} \sim 3 C$. Since $\left|K_{S}\right|$ cuts on the general curve in $|C|$ a $g_{3}^{1}$ without base points, this curve is not hyperelliptic.

Claim 3: if $p_{g}=4$, then either $S$ contains a rational pencil $|C|$ with $C^{2}=$ $1, K_{S} C=3$, such that the general curve of $|C|$ is irreducible and nonhyperelliptic, or $K_{S}^{2}=8$ and $S$ contains a rational pencil $|C|$ with $C^{2}=$ $2, K_{S} C=4$, such that the general curve of $|C|$ is irreducible and nonhyperelliptic.

We start by showing that $\Sigma$ is the quadric cone. Assume on the contrary that $\Sigma$ is a smooth quadric, and denote by $D$ the pull-back of a general tangent section of $\Sigma$. One can write $D=A+B$, where $A$ and $B$ are pull-backs of lines on $\Sigma$. Since the general canonical curve is smooth by assumption, and thus in particular $\left|K_{S}\right|$ has no fixed part, $D$ is a canonical curve. Notice also that $A^{2}, B^{2} \geq 0$ since they move in linear systems without fixed components, and $A B \geq 3$. On the other hand, $A B=A\left(K_{S}-A\right)$ is even and therefore $A B \geq 4$. This implies that $\left|K_{S}\right|$ has base points and that there exists a base point $Q$ of $\left|K_{S}\right|$ that is also a base point for both $|A|$ and $|B|$. So $A^{2}, B^{2}>0$ and $K_{S}^{2}=A^{2}+B^{2}+2 A B \geq 10$, contradicting Claim 1. We conclude that $\Sigma$ is a quadric cone.

As in the proof of Claim 2, consider the pull-back $D$ of a hyperplane section of $\Sigma$ passing through the vertex and write $D=2 C+Z$, where $C$ is the pull-back of a ruling of the cone and $Z$ is an effective divisor, possibly zero, that is contracted to the vertex. Notice that $C^{2} \geq 0$, as $C$ moves in a linear system without fixed components, and $2 C^{2} \leq K_{S} C$. One has $K_{S} C=D C \geq 3$ and $2 C K_{S} \leq K_{S}^{2} \leq 9$, so that either $K_{S} C=3$ or $K_{S} C=4$. In the former case, $C^{2}$ is odd and thus, by the above discussion, $C^{2}=1$. Again (as in the proof of the preceding claim) it is easy to see that the general curve in $|C|$ is non hyperelliptic and so $S$ contains a genus 3 pencil as stated.

On the other hand, if $K_{S} C=4$ then $\left|K_{S}\right|$ has base points and there exists a base point $Q$ of $\left|K_{S}\right|$ that is also a base point of $|C|$, and so $C^{2}>0$. So we conclude that $C^{2}=2$ and, by the index theorem, that $K_{S}^{2} \leq 8$. But $K_{S}^{2} \geq 2 K_{S} C \geq 8$, so $K_{S}^{2}=8$. Using the same argument as in the previous claim it is easy to see that the general curve in $|C|$ is not hyperelliptic. $\diamond$ 


\section{Surfaces with a special pencil $I$}

In this section we study in detail the class of the surfaces containing a pencil $C$, with $C^{2}=1, K_{S} C=3$, to which one of the types of surfaces found in section 2 belongs. So, throughout this section, we make the following assumption:

Assumption 3.1 $S$ is a minimal surface of general type with $p_{g} \geq 4$ containing a positive-dimensional linear system $|C|$ with $K_{S} C=3, C^{2}=1$, and such that the general curve in $|C|$ is irreducible, non-hyperelliptic.

We describe the possible invariants of such surfaces, show that the canonical system of these surfaces contains a smooth curve and finally establish some properties that will enable us to construct examples of all these surfaces. In particular we prove in this section the following:

Theorem 3.2 Let $S$ be a surface satisfying assumption 3.1. Then

i) $q=0$ and either $p_{g}=5$ and $K_{S}^{2}=9$, or $p_{g}=4$ and $6 \leq K_{S}^{2} \leq 8$;

ii) $|C|$ is a pencil with a simple base point $P$;

iii) $\operatorname{deg} \phi_{K}=3$;

iv) the general curve in $\left|K_{S}\right|$ is smooth;

$v)$ the linear system $|3 C|$ defines a morphism $S \rightarrow \mathbf{P}^{4}$ of degree 3 onto the normal rational cubic cone.

To prove theorem 3.2 we will need various facts, that we now establish.

Lemma 3.3 If $S$ is a surface as in 3.1, then $|C|$ is a pencil with a simple base point.

Proof: Remark first of all that $|C|$ has no fixed components, since it is positive dimensional and the general curve of $|C|$ is irreducible by assumption. So, if $\operatorname{dim}|C|$ were greater than 1 , then $|C|$ would be base point free, and therefore a general 2-dimensional subsystem of $|C|$ would define an isomorphism of $S$ with $\mathbf{P}^{2}$. So $|C|$ is necessarily 1-dimensional and therefore it has a simple base point. $\diamond$

Notation 3.4 We denote by $C$ a general element of $|C|$ and by $P$ the base point of the pencil $|C|$. 
Lemma 3.5 If $S$ is a surface as in assumption 3.1, then $K_{S}^{2} \leq 9$ and $K_{S}^{2}=9$ if and only if $K_{S} \sim 3 C$. Furthermore $\left|K_{S}\right|$ is not composed with $|C|$.

Proof: By the index theorem, the conditions $C^{2}=1, K_{S} C=3$ imply $K_{S}^{2} \leq 9$, with equality holding if and only if $K_{S} \sim 3 C$. By proposition (1.7) of CFM, if $\left|K_{S}\right|$ is composed with $|C|$ then $K_{S}^{2}=9, p_{g}=4$ and $\left|K_{S}\right|=|3 C|$. But this contradicts [Z], section 2.6.2. $\diamond$

Lemma 3.6 If $S$ is a surface as in assumption 3.1, then:

i) $p_{g}=4$ or $p_{g}=5$, and $p_{g}=5$ iff $K_{S} \equiv 3 C$;

ii) $h^{0}\left(S, \mathcal{O}_{S}\left(K_{S}-n C\right)\right)=p_{g}-n-1$, for $n=1,2,3$;

iii) if $C$ is general, then $\left|K_{S}\right|_{C}$ is a complete and base point free linear system of dimension 2 .

Proof: Lemma 3.5 yields $K_{S}\left(K_{S}-3 C\right) \leq 0$, with equality holding if and only if $K_{S} \sim 3 C$. Thus $h^{0}\left(S, \mathcal{O}_{S}\left(K_{S}-3 C\right)\right) \leq 1$, with equality holding if and only $K_{S} \equiv 3 C$.

Consider the restriction maps

$$
r_{n}: H^{0}\left(S, \mathcal{O}_{S}\left(K_{S}-n C\right)\right) \rightarrow H^{0}\left(C, \mathcal{O}_{C}\left(K_{S}-n C\right)\right)
$$

and notice that ker $r_{n} \simeq H^{0}\left(S, \mathcal{O}_{S}\left(K_{S}-(n+1) C\right)\right)$. Since $K_{S} C=3$, one has $h^{0}\left(C, \mathcal{O}_{C}\left(K_{S}\right)\right) \leq 2$; on the other hand, since $\left|K_{S}\right|$ is not composed with $|C|$ by lemma 3.5, one has $\operatorname{dim} \operatorname{Im} r_{0} \geq 2$. Thus $h^{0}\left(C, \mathcal{O}_{C}\left(K_{S}\right)=2\right.$, the map $r_{0}$ is onto and $h^{0}\left(S, \mathcal{O}_{S}\left(K_{S}-C\right)\right)=p_{g}-2$. Now $\left(K_{S}-C\right) C=2$, hence $\operatorname{dim} \operatorname{Im} r_{1}=h^{0}\left(C, \mathcal{O}_{C}\left(K_{S}-C\right)=1\right.$, because $K_{S}-C$ is effective, and $C$ is non-hyperelliptic by assumption. Thus $h^{0}\left(S, \mathcal{O}_{S}\left(K_{S}-2 C\right)\right)=p_{g}-3$. Similarly, one gets $h^{0}\left(S, \mathcal{O}_{S}\left(K_{S}-3 C\right)\right)=p_{g}-4$. From $h^{0}\left(S, \mathcal{O}_{S}\left(K_{S}-3 C\right)\right) \leq$ 1 we have then that $p_{g} \leq 5$ and $p_{g}=5$ iff $K_{S}-3 C \equiv 0$. From the above analysis it follows in particular that $\left|K_{S}\right|_{C}$ is a complete linear system of dimension 2 and degree 3 . Since $C$ is not hyperelliptic, $\left|K_{S}\right|_{C}$ has no fixed point. $\diamond$

Lemma 3.7 If $S$ is a surface as in assumption 3.1 and $p_{g}=4$, then $6 \leq$ $K_{S}^{2} \leq 8$, and $K_{S} \equiv 2 C+Z$, where $Z$ is a 2-connected curve with $Z C=1$, $Z K_{S}=K_{S}^{2}-6$ and $Z^{2}=K_{S}^{2}-8$. Moreover, if $K_{S}^{2}=6$ then $Z$ is a smooth rational curve; if $K_{S}^{2}=7,8$ then $Z$ contains the base point $P$ of $|C|$ and there exists $C^{\prime} \in|C|$ such that $Z \subset C^{\prime}$. 
Proof: Suppose $p_{g}=4$. By lemma 3.6 ii), $h^{0}\left(S, \mathcal{O}_{S}\left(K_{S}-2 C\right)\right)=1$, and hence $K_{S} \equiv 2 C+Z$, where $Z$ is an effective divisor. One has $Z C=1$, since $K_{S} C=3$, and $K_{S}^{2}=2 K_{S} C+K_{S} Z \geq 6$, since $K_{S}$ is nef. It follows immediately that $Z^{2}=K_{S}^{2}-8$ and $K_{S} Z=K_{S}^{2}-6$. In particular, remark that if $K_{S}^{2}=9$ then $Z \sim C$ by lemma 3.5.

Let us notice that $Z$ is 2 -connected. In fact assume otherwise. Then $Z$ decomposes as $Z=A+B$ with $A B \leq 1$. Since canonical divisors are 2 -connected, one has $2 \leq A\left(K_{S}-A\right)=A B+2 A C$ and thus $A C \geq 1$. Similarly we have $B C \geq 1$, but this contradicts $Z C=1$. Remark that if $Z^{2}=-2, p_{a}(Z)=0$ and therefore 2-connectedness of $Z$ implies that $Z$ is an irreducible curve. Now let us see that if $K_{S}^{2} \geq 7$ then the base point $P$ of $|C|$ lies on $Z$. In fact, in this case $Z$ is a 2-connected curve with $p_{a}(Z) \geq 1$ and hence $h^{0}\left(Z, \mathcal{O}_{Z}(C)\right)=1$ (see [CFM], (A.5)). This implies that $|C|$ has a base point lying on $Z$ and thus $P \in Z$. Since $P$ lies on $Z$, the restriction map

$$
r: H^{0}\left(S, \mathcal{O}_{S}(C)\right) \rightarrow H^{0}\left(Z, \mathcal{O}_{Z}(C)\right)
$$

has 1-dimensional image, and therefore there is a curve $C^{\prime} \in|C|$ such that $C^{\prime}=Z+\Delta$, with $\Delta \geq 0$. Now if $K_{S}^{2}=9$, then $Z \sim C$ implies that $\Delta=0$ and $Z \equiv C$. Therefore, this case does not occur by lemma 3.6, i). $\diamond$

Lemma 3.8 If $S$ is a surface as in assumption 3.1, then the canonical map $\phi_{K}$ of $S$ maps $S$ 3-1 onto the rational normal cone in $\mathbf{P}^{p_{g}-1}$. The curves of $|C|$ are mapped 3-1 onto the rulings of the cone and the base point $P$ is mapped to the vertex. The map $\phi_{K}$ is a morphism iff $K_{S}^{2}=6$ or $K_{S}^{2}=9$.

Proof: Remark that by lemma 3.6, iii), $\phi_{K}$ maps a general $C 3-1$ onto a line. Moreover $\phi_{K}$ separates the curves of $|C|$, and thus $\operatorname{deg} \phi_{K}=3$.

Let $\Sigma$ be the canonical image of $S$ and let $d=\operatorname{deg} \Sigma$; one has:

$$
K_{S}^{2} \geq \operatorname{deg} \phi_{K} d=3 d \geq 3\left(p_{g}-2\right)
$$

By lemmas 3.6, 3.7 either one has $K_{S}^{2}=9, p_{g}=5$, or $6 \leq K_{S}^{2} \leq 8, p_{g}=4$. In the former case, (3.1) implies $d=3$ and $\left|K_{S}\right|$ base point free; in the latter case, it implies $d=2$ and $\left|K_{S}\right|$ is base point free iff $K_{S}^{2}=6$. So $\Sigma$ is a surface of minimal degree, ruled by the images of the curves of $|C|$. To show that $\Sigma$ is actually a cone it is enough to remark that, by lemma 3.6, iii), $P$ is not a base point of $\phi_{K}$ and so the image lines of the curves of $|C|$ all go through the point $\phi_{K}(P)$. $\diamond$ 
Lemma 3.9 If $S$ is a surface as in assumption 3.1, then $q=0$.

Proof: Let us remark first that $q \leq 1$. Indeed notice that, since $C^{2}=1$, $h^{0}\left(C, \mathcal{O}_{C}(C)\right)=1$ and the restriction map $H^{0}\left(S, \mathcal{O}_{S}(C)\right) \rightarrow H^{0}\left(C, \mathcal{O}_{C}(C)\right)$ is onto. Furthermore, by lemma 3.6, ii), $h^{0}\left(C, \mathcal{O}_{C}\left(K_{S}\right)\right)=2$ and the restriction map $H^{0}\left(S, \mathcal{O}_{S}\left(K_{S}\right)\right) \rightarrow H^{0}\left(C, \mathcal{O}_{C}\left(K_{S}\right)\right)$ is also surjective. So we can choose $t_{0}, t_{1} \in H^{0}\left(S, \mathcal{O}_{S}\left(K_{S}\right)\right)$ and $s \in H^{0}\left(S, \mathcal{O}_{S}(C)\right)$, such that the images of $t_{0}, t_{1}$ generate $H^{0}\left(C, \mathcal{O}_{C}\left(K_{S}\right)\right)$ and the image of $s$ generates $H^{0}\left(C, \mathcal{O}_{C}(C)\right)$. Then $s t_{0}, s t_{1}$ map to linearly independent sections of $H^{0}\left(C, \mathcal{O}_{C}\left(K_{S}+C\right)\right)=H^{0}\left(C, \omega_{C}\right)$. Consider now the exact sequence:

$$
0 \rightarrow \mathcal{O}_{S}\left(K_{S}\right) \rightarrow \mathcal{O}_{S}\left(K_{S}+C\right) \rightarrow \omega_{C} \rightarrow 0
$$

Since $C^{2}=1$ and $C$ is irreducible, we have $h^{1}\left(S, \mathcal{O}_{S}\left(K_{S}+C\right)\right)=0$ (see [B] pg. 178), and the long cohomology sequence yields:

$$
H^{0}\left(S, \mathcal{O}_{S}\left(K_{S}+C\right)\right) \rightarrow H^{0}\left(C, \omega_{C}\right) \rightarrow H^{1}\left(S, \mathcal{O}_{S}\left(K_{S}\right) \rightarrow 0 .\right.
$$

Hence $q=h^{1}\left(S, \mathcal{O}_{S}\left(K_{S}\right)\right) \leq 1$.

If $q=1$, then the inequality $K_{S}^{2} \geq 2 p_{g}$ (see [Dd, Th. 6.1) and lemma 3.7 leave us with the case $p_{g}=4, K_{S}^{2}=8$. Then $\chi\left(\mathcal{O}_{S}\right)=4$ and $K_{S}^{2}<\frac{8}{3} \chi\left(\mathcal{O}_{S}\right)$. By [Ho3], then the Albanese pencil is a genus 2 pencil. But this contradicts lemma 3.8, because the canonical map of a surface with a genus 2 pencil has even degree. $\diamond$

Lemma 3.10 If $S$ is a surface as in assumption 3.1, then $h^{1}\left(S, \mathcal{O}_{S}(3 C)\right)=$ $0, h^{0}\left(S, \mathcal{O}_{S}(3 C)\right)=5$ and $|3 C|$ is base point free.

Proof: Notice first that the image of the restriction map $H^{0}\left(S, \mathcal{O}_{S}(3 C)\right) \rightarrow$ $H^{0}\left(C, \mathcal{O}_{C}(3 C)\right)$ has dimension at most 2 and the image of $H^{0}\left(S, \mathcal{O}_{S}(2 C)\right) \rightarrow$ $H^{0}\left(C, \mathcal{O}_{C}(2 C)\right)$ has dimension 1 , since $C$ is not hyperellliptic; so, arguing as in the proof of lemma 3.6, it is easy to show that $h^{0}\left(S, \mathcal{O}_{S}(3 C)\right) \leq 5$. If $p_{g}=5$, then by lemma 3.6, i), $h^{0}\left(S, \mathcal{O}_{S}(3 C)\right)=p_{g}=5$. If $p_{g}=4$, then $h^{2}\left(S, \mathcal{O}_{S}(3 C)\right)=h^{0}\left(S, \mathcal{O}_{S}\left(K_{S}-3 C\right)\right)=0$ by lemma 3.6, ii). Thus the Riemann-Roch theorem yields $h^{0}\left(S, \mathcal{O}_{S}(3 C)\right)=\chi\left(\mathcal{O}_{S}\right)+h^{1}\left(S, \mathcal{O}_{S}(3 C)\right)=$ $5+h^{1}\left(S, \mathcal{O}_{S}(3 C)\right)$. (Recall that $q=0$ by lemma 3.9). So we conclude that $h^{0}\left(S, \mathcal{O}_{S}(3 C)\right)=5, h^{1}\left(S, \mathcal{O}_{S}(3 C)\right)=0$ and $|3 C|_{C}$ is equal to the complete linear system $|3 P|$.

Finally, observe that the only possible base point of $|3 C|$ is the base point $P$ of $|C|$; on the other hand if $P$ were a base point of $|3 C|$, then the moving part of the system $|3 C|_{C}$ would be a $g_{2}^{1}$ and $C$ would be hyperelliptic. $\diamond$ 
Lemma 3.11 If $S$ is a surface as in assumption 3.1, then the linear system $|3 C|$ defines a morphism $f: S \rightarrow \mathbf{P}^{4}$ of degree 3 onto the rational normal cubic cone $C_{3}$. The curves of $|C|$ are the pull-backs via $f$ of the rulings of $C_{3}$ and the point $P$ is mapped to the vertex.

Proof: Since $C^{2}=1$ and $|3 C|$ is base point free by lemma 3.10, every curve of $|C|$ is mapped by $f 3$-to- 1 onto a line through the point $f(P)$. So the image of $S$ is the rational cubic cone and $f$ has degree 3 . $\diamond$

Remark 3.12 If $p_{g}=5$, then the morphisms $f$ and $\phi_{K}$ coincide by lemma 3.6, i). If $p_{g}=4$ and $P \in Z$ (see lemma 3.7), then $\left|K_{S}\right| \subset|3 C|$ and $\phi_{K}$ is the composition of $f$ with projection from a point of $C_{3}$. Finally, if $K_{S}^{2}=6$, $p_{g}=4$ and $P \notin Z$, then the systems $|3 C|$ and $\left|K_{S}\right|$ restrict to different linear systems on a general $C$ and therefore $f$ and $\phi_{K}$ are not related by a birational transformation of the cones preserving the rulings.

To finish the proof of thm. 3.2, we show that for these surfaces the general curve in $\left|K_{S}\right|$ is smooth and therefore they satisfy the hypothesis of theorem 2.1.

Lemma 3.13 If $S$ is a surface as in assumption 3.1, then the general curve in $\left|K_{S}\right|$ is smooth.

Proof: For $K_{S}^{2}=9,6$, this is immediate since $\left|K_{S}\right|$ is base point free by lemma 3.8. For the other cases $\left(K_{S}^{2}=7,8\right)$ the canonical map is $3-1$ onto the quadric cone. If $\left|K_{S}\right|$ has no fixed part, then its base locus is a zerodimensional scheme of length $\leq 2$, and therefore it is smooth: thus the general canonical curve is smooth by Bertini's theorem. Therefore assume that $\left|K_{S}\right|$ has a fixed part $F$ and write $K_{S}=M+F$. One has: $M^{2} \geq 6$, since the linear system $|M|$ maps $S 3-1$ onto the quadric cone, and $M F \geq 2$, since canonical divisors are 2-connected. Moreover the canonical divisor of a minimal surface is nef, and thus we have: $8 \geq K_{S}^{2}=K_{S} M+K_{S} F \geq$ $K_{S} M=M^{2}+M F \geq 8$, and thus the only possibility is $K_{S}^{2}=8, K_{S} F=0$, $M^{2}=6$ and $F^{2}=-2$. Therefore $|M|$ is base point free, and every irreducible component $\theta$ of $F$ is a smooth rational curve with self intersection -2 , i.e a $(-2)$-curve. For any such $\theta$ we have $\theta C=0$, since $C$ is nef and $C F=0$, and $\theta Z \geq 0$ since $Z$ is $2-$ connected by lemma 3.7 .

Consider the effective divisor $\Gamma$ such that $\Gamma$ is linearly equivalent to $C-Z$ (see lemma 3.7). One has $\Gamma Z=1$ and $\Gamma C=0$, hence $\Gamma^{2}=-1$, 
$K_{S} \Gamma=1$. Furthermore $\Gamma$ is 2-connected. In fact, assume otherwise. Then we can write $\Gamma=A+B$ where $A, B$ are effective non-zero divisors such that $A B \leq 1, A C=B C=0$. Since $C=\Gamma+Z$ and $K_{S}=2 C+Z$, we can write $K_{S}=A+B+C+2 Z$ : if, for instance, $A Z=0$ then the divisors $A$ and $C+2 Z+B$ give a decomposition of $K_{S}$ contradicting the fact that $K_{S}$ is 2 -connected. So we have $A Z>0$ and, by the same argument, $B Z>0$, contradicting $\Gamma Z=1$. Therefore $\Gamma$ is 2 -connected, hence for any irreducible component $\theta$ of $F, \theta \Gamma \geq 0$. Since $\theta C=\theta(Z+\Gamma)=0$, we have then necessarily $\theta \Gamma=\theta Z=0$ and therefore $F \Gamma=0$. But then $M \Gamma=1$, and so by proposition (A.5) of [CFM]), the restriction map

$$
r: H^{0}\left(S, \mathcal{O}_{S}(M)\right) \rightarrow H^{0}\left(\Gamma, \mathcal{O}_{\Gamma}(M)\right)
$$

has 1-dimensional image, a contradiction since $|M|$ is base point free.

Therefore $\left|K_{S}\right|$ has no fixed components and thus we proved the lemma. $\diamond$

Proof of Theorem 3.2: The theorem follows directly from lemmas $3.3, \ldots$, 3.13. $\diamond$

We continue this section by establishing some facts that will enable us to construct examples of surfaces satisfying assumption 3.1 and having all the possible values of the invariants.

Lemma 3.14 Let $S$ be a surface as in assumption 3.1; then

i) if $K_{S}^{2} \geq 7$ or $K_{S}^{2}=6$ and $P \in Z$ (see lemma 3.7), then $h^{0}\left(S, \mathcal{O}_{S}(n C)\right)=$ $6+n(n-3) / 2$ for $n \geq 4, P$ is a simple base point of $|5 C|$, and $|n C|$ is base point free for $n=4$ and $n \geq 6$;

iii) if $K_{S}^{2}=6$ and $P \notin Z$ (see lemma 3.7), then $h^{0}\left(S, \mathcal{O}_{S}(n C)\right)=$ $5+n(n-3) / 2$ for $n \geq 4, P$ is a simple base point of $|4 C|$, and $|n C|$ is base point free for $n \geq 5$.

Proof:

Case i):

Assume first that $p_{g}(S)=5$. By lemma 3.6, for $n \geq 4 n C$ is the adjoint of a nef and big divisor, so by Kawamata-Viehweg vanishing $h^{0}\left(S, \mathcal{O}_{S}(n C)\right)=$ $\chi\left(\mathcal{O}_{S}(n C)\right)$ can be computed by Riemann-Roch.

Assume now that $p_{g}(S)=4$. By lemma 3.7, $K_{S}\left(K_{S}-n C\right)=K_{S}^{2}-3 n \leq$ $8-3 n<0$, and thus $0=h^{0}\left(S, \mathcal{O}_{S}\left(K_{S}-n C\right)\right)=h^{2}\left(S, \mathcal{O}_{S}(n C)\right)$ for $n \geq 3$. So we have:

$$
h^{0}\left(S, \mathcal{O}_{S}(n C)\right)=5+n(n-3) / 2+h^{1}\left(S, \mathcal{O}_{S}(n C)\right), \quad n \geq 3 .
$$


Denote by $C$ a smooth element of $|C|$ and consider the sequence:

$$
0 \rightarrow \mathcal{O}_{S} \rightarrow \mathcal{O}_{S}(C) \rightarrow \mathcal{O}_{C}(P) \rightarrow 0
$$

Twisting (3.3) by $3 C$ and recalling that $h^{1}\left(S, \mathcal{O}_{S}(3 C)\right)=0$ by lemma 3.10 , one obtains exact sequences:

$$
0 \rightarrow H^{0}\left(S, \mathcal{O}_{S}(3 C)\right) \rightarrow H^{0}\left(S, \mathcal{O}_{S}(4 C)\right) \rightarrow H^{0}\left(C, \mathcal{O}_{C}(4 P)\right) \rightarrow 0
$$

and $0 \rightarrow H^{1}\left(S, \mathcal{O}_{S}(4 C)\right) \rightarrow H^{1}\left(C, \mathcal{O}_{C}(4 C)\right) \rightarrow 0$. By the adjunction formula, $4 P$ is a canonical divisor on $C$ and thus $h^{1}\left(S, \mathcal{O}_{S}(4 C)\right)=1$, $h^{0}\left(S, \mathcal{O}_{S}(4 C)\right)=7$. Twisting $(3.3)$ by $n C, n \geq 4$, and passing to the associate cohomology sequence one obtains a surjection $H^{1}\left(S, \mathcal{O}_{S}(n C)\right) \rightarrow$ $H^{1}\left(S, \mathcal{O}_{S}((n+1) C)\right)$, since $h^{1}\left(C, \mathcal{O}_{C}((n+1) P)\right)=0$. So we have $h^{1}\left(S, \mathcal{O}_{S}(n C)\right) \leq$ 1 for $n \geq 4$. By Serre duality, $h^{1}\left(S, \mathcal{O}_{S}(n C)\right)=h^{1}\left(S, \mathcal{O}_{S}(-(n-3) C-\right.$ $\Gamma)$ ), where $\Gamma$ is the only effective divisor linearly equivalent to $C-Z$ (cf. lemma 3.7). Since $\Gamma C=0$, if $D$ is a general element of $|(n-3) C|$ then $D+\Gamma$ is a disconnected curve and $h^{0}\left(D+\Gamma, \mathcal{O}_{D+\Gamma}\right) \geq 2$. This implies that $h^{1}\left(S, \mathcal{O}_{S}(n C)\right)=h^{1}\left(S, \mathcal{O}_{S}(-(n-3) C-\Gamma)\right) \geq 1$ and so, eventually, $h^{1}\left(S, \mathcal{O}_{S}(n C)\right)=1$ for $n \geq 4$. So $h^{0}\left(S, \mathcal{O}_{S}(n C)\right)$ can now be computed from (3.2). Using again Riemann-Roch on the curve $C$, one sees that $|5 P|=$ $P+|4 P|$ and thus $P$ is a base point of $|5 C|$. It is a simple base point (and the only one), since $|4 C|$ is free and $P$ is the only base point of $|C|$. We have shown above that for $n \geq 4$ the map $H^{1}\left(S, \mathcal{O}_{S}(n C)\right) \rightarrow H^{1}\left(S, \mathcal{O}_{S}((n+1) C)\right)$ is an isomorphism; it follows that $H^{0}\left(S, \mathcal{O}_{S}(n C)\right) \rightarrow H^{0}\left(C, \mathcal{O}_{C}(n P)\right)$ is surjective for $n \geq 5$ and thus $|n C|$ is free for $n \geq 6$.

Case ii):

The arguments here are analogous to those used in the proof of case i). Notice that $K_{S}\left(K_{S}-n C\right)=6-3 n<0$ for $n \geq 3$, so that $h^{2}\left(S, \mathcal{O}_{S}(n C)\right)=$ $h^{0}\left(S, \mathcal{O}_{S}\left(K_{S}-n C\right)=0\right.$ for $n \geq 3$. Fix a smooth $C \in|C|$ and denote by $Q$ the intersection point of $C$ and $Z$. We have $P \neq Q$ by assumption. By the adjunction formula, $3 P+Q$ is a canonical divisor on $C$ and so, using Riemann-Roch on $C$, one shows easily that $|4 P|=P+|3 P|$. Sequence (3.3) and the fact that, by lemma 3.10, $h^{1}\left(S, \mathcal{O}_{S}(3 C)\right)=0$ imply that $P$ is a base point of $|4 C|, h^{0}\left(S, \mathcal{O}_{S}(4 C)\right)=7$ and $h^{1}\left(S, \mathcal{O}_{S}(4 C)\right)=0$. Since $|3 C|$ is free and $P$ is a simple base point of $|C|, P$ is also a simple base point of $|4 C|$. Furthermore, for $n \geq 4$ the map $H^{1}\left(S, \mathcal{O}_{S}(n C)\right) \rightarrow H^{1}\left(S, \mathcal{O}_{S}((n+1) C)\right)$ is onto, since $H^{1}\left(C, \mathcal{O}_{C}((n+1) P)\right)=0$. So $h^{1}\left(S, \mathcal{O}_{S}(n C)\right)=0$ for $n \geq 4$, $H^{0}\left(S, \mathcal{O}_{S}(n C)\right) \rightarrow H^{0}\left(C, \mathcal{O}_{C}(n P)\right)$ is onto for $n \geq 5$ and thus $|n C|$ is free for $n \geq 4$. Finally, $h^{0}\left(S, \mathcal{O}_{S}(n C)\right)$ can now be computed by means of the Riemann-Roch formula. $\diamond$ 


\section{Surfaces with a special pencil $I I$}

In this section we study the surfaces containing a positive-dimensional linear system $|C|$ satisfying $K_{S} C=4, C^{2}=2$ and such that the general curve in $|C|$ is irreducible, non-hyperelliptic, to which belong one of the types of surfaces we encountered in section 2 .

So, throughout this section, we make the following assumption

Assumption 4.1 $S$ is a minimal surface of general type with $p_{g} \geq 4$, containing a positive-dimensional linear system $|C|$ satisfying $K_{S} C=4, C^{2}=$ 2 , and such that the general curve in $|C|$ is irreducible, non-hyperelliptic.

Notation 4.2 We will denote by $C$ a general element of $|C|$.

We want to prove the following theorem and also to establish some properties that will enable us to construct examples.

Theorem 4.3 Let $S$ be a surface as in assumption 4.1. Then

i) $K_{S}^{2}=8, p_{g}=4$ and $q=0$;

ii) $|C|$ is a pencil with two base points $P$ and $P_{2} ; K_{S}=2 C$ and the general curve of $K_{S}$ is smooth;

iii) $\operatorname{deg} \phi_{K}=3$ or $\operatorname{deg} \phi_{K}=4$;

iv) if $\operatorname{deg} \phi_{K}=3, P_{2}$ is infinitely near to $P$; if we denote by $S^{\prime}$ the blowup of $S$ at $P$ and by $C^{\prime}$ the strict transform of $C$ on $S^{\prime}$, then the linear system $\left|3 C^{\prime}\right|$ defines a morphism $S \rightarrow \mathbf{P}^{4}$ of degree 3 onto the normal rational cubic cone.

To prove the theorem we will need the following:

Lemma 4.4 If $S$ is a surface as in assumption 4.1, then $|C|$ is a pencil, $p_{g}=4, K_{S}^{2}=8, K_{S}=2 C, \phi_{K}$ is generically finite, $\operatorname{deg} \phi_{K}=3$ or 4 and the general curve of $\left|K_{S}\right|$ is smooth .

Proof: Notice first that the assumptions imply that the general curve in $|C|$ is smooth irreducible of genus 4. Moreover, if $\operatorname{dim}|C|>1$, then the moving part of the restriction of $|C|$ to a general $|C|$ would be a $g_{2}^{1}$ and $C$ would be hyperelliptic. So $|C|$ is a pencil. For a general $C \in|C|$, one has, by Riemann-Roch: $h^{0}\left(C, \mathcal{O}_{C}\left(K_{S}\right)\right)=h^{0}\left(C, \mathcal{O}_{C}(C)\right)+1=2$, the last equality holding because $C$ is not hyperelliptic. Now, arguing as in the proof of lemma 3.6, one shows that $h^{0}\left(S, K_{S}-2 C\right) \geq p_{g}-3 \geq 1$. So, 
$K_{S}^{2}-8=K_{S}\left(K_{S}-2 C\right) \geq 0$, since $K_{S}$ is nef. On the other hand, the index theorem gives $K_{S}^{2} \leq 8$, with equality holding if and only if $K_{S} \sim 2 C$. Since we have shown that $K_{S}-2 C$ is effective, we conclude that $K_{S}=2 C$ and $K_{S}^{2}=8$. In particular, $1=h^{0}\left(S, \mathcal{O}_{S}\left(K_{S}-2 C\right)\right) \geq p_{g}-3 \geq 1$ and so $p_{g}=4$.

The image of $\phi_{K}$ is a surface, since otherwise $\phi_{K}$ would be composed with $C$ and thus $p_{g}(S)=3$. The assertion about the degree of $\phi_{K}$ can be proven exactly as in lemma 3.8 and the last assertion is obvious from $K_{S}=2 C . \diamond$

Lemma 4.5 If $S$ is a surface as in assertion 4.1, then $q=0$.

Proof: This lemma is proved for a slightly different situation in CFM, proposition (3.5). For the reader's convenience we give here an outline of the proof.

First of all, arguing as in the proof of lemma 3.9 one shows that $q \leq 2$. Next assume that $q \neq 0$ and let $\mu$ be an element in $\operatorname{Pic}^{0}(S)$. From the exact sequence

$$
0 \rightarrow \mu \rightarrow \mu(C) \rightarrow \mathcal{O}_{C}(C) \otimes \mu \rightarrow 0
$$

we see that, for $\mu$ general, one has $h^{0}(S, \mu(C)) \leq 1$, because $C^{2}=2$. By the same reason, also $h^{0}\left(S, \mu^{\vee}(C)\right) \leq 1$. By Serre duality and lemma 4.4, it follows $h^{2}(S, \mu(C))=h^{0}\left(S, \mu^{\vee}(C)\right.$ and thus, by the Riemann-Roch theorem, one has:

$$
2 \geq h^{0}(S, \mu(C))+h^{0}\left(S, \mu^{\vee}(C)\right)=p_{g}-q+h^{1}(S, \mu(C)) \geq p_{g}-q
$$

Since $p_{g}=4$ and $q \leq 2$, the above inequality yields $q=2$ and $h^{0}(S, \mu(C))=$ $h^{0}\left(S, \mu^{\vee}(C)\right)=1$.

So we can define a rational map $\rho: \operatorname{Pic}^{0}(S) \rightarrow C^{(2)}$, which sends a general element $\mu$ to the only effective divisor in $\left|\mathcal{O}_{C}(C) \otimes \mu\right|$. The map $\rho$ is generically injective. Since $C$ is a smooth curve of genus 4 , we find a contradiction, because the surface of general type $C^{(2)}$ cannot be dominated by the abelian surface $P i c^{0}(S)$. $\diamond$

Lemma 4.6 Let $S$ be a surface as in assumption 4.1, and assume that $\operatorname{deg} \phi_{K}=3$; then:

i) the image of $\phi_{K}$ is a quadric cone;

ii) the base locus of $\left|K_{S}\right|$ consists of a point $P$ and a point $P_{1}$, infinitely near to $P$ and in particular the general canonical curve is smooth;

iii) the base locus of $|C|$ consists of $P$ and a point $P_{2}$ infinitely near to $P$, with $P_{2} \neq P_{1}$. 
Proof: Since $K_{S} \equiv 2 C,\left|K_{S}\right|$ has no fixed component. Denote by $d$ the degree of the image $\Sigma$ of $\phi_{K}$; one has $8=K_{S}^{2} \geq d \operatorname{deg} \phi_{K}=3 d \geq 3\left(p_{g}-2\right)=$ 6 . So $d=2$ is the only possibility and $\Sigma$ is a singular quadric, since $K_{S}=2 C$. Moreover the above inequality implies that $\left|K_{S}\right|$ has two simple base points $P$ and $P_{1}$, with $P_{1}$ possibly infinitely near to $P$. Notice that by Bertini's theorem this implies that the general canonical curve is smooth. We wish to show that $P_{1}$ is actually infinitely near to $P$. So assume otherwise: then both $P$ and $P_{1}$ are base points of $|C|$ and thus also of $\left|K_{S}\right|_{C}$. This implies that the moving part of $\left|K_{S}\right|_{C}$ is a $g_{2}^{1}$ and $C$ is hyperelliptic, against the assumptions. Notice that $P$ is necessarily a base point of $C$; since $C^{2}=2$, $C$ has also another base point $P_{2}$. We are going to prove that $P_{2}$ is also infinitely near to $P$ but $P_{2} \neq P_{1}$. By adjunction, the sheaf $\mathcal{O}_{S}\left(2 K_{S}\right)$ restricts to $\omega_{C}\left(P+P_{2}\right)$ on $C$; by Riemann-Roch on $C$, this implies that $\left|2 K_{S}\right|$ does not separate $P$ and $P_{2}$, and thus also $\left|K_{S}\right|$ does not separate $P$ and $P_{2}$. If $P_{2}$ is not infinitely near to $P$, this is equivalent to saying that $P_{2}$ is also a base point of $\left|K_{S}\right|$, contradicting what we have just proven. So we conclude that $P_{2}$ is infinitely near to $P$. Finally, if $P_{1}$ and $P_{2}$ were equal, then again the moving part of $\left|K_{S}\right|_{C}$ would be a $g_{2}^{1}$, and $C$ would be hyperelliptic. $\diamond$

Notation 4.7 We denote by $\epsilon^{\prime}: S^{\prime} \rightarrow S$ the blow-up of $S$ at the common base point $P$ of $|C|$ and $\left|K_{S}\right|$, by $E^{\prime}$ the exceptional curve of $\epsilon^{\prime}$, again by $C$ the pull-back of $C$ to $S^{\prime}$ and by $C^{\prime}$ the divisor $C-E^{\prime}$.

Lemma 4.8 Let $S$ be a surface as in assumption 4.1 such that $\operatorname{deg} \phi_{K}=3$, and let $S^{\prime}, C^{\prime}$ be as in notation 4.7; then $h^{0}\left(S^{\prime}, \mathcal{O}_{S^{\prime}}\left(3 C^{\prime}\right)\right)=5$ and the system $\left|3 C^{\prime}\right|$ is base point free.

Proof: By lemma 4.6, the moving part of the canonical system of $S^{\prime}$ is $|M|=\left|2 C^{\prime}+E^{\prime}\right|$. Let $C^{\prime} \in\left|C^{\prime}\right|$ be a general curve: then $|M|_{C^{\prime}}=\left|3 P_{2}\right|$ is a base point free complete linear system of dimension 1 and degree 3, since the canonical map has degree 3 . Using restriction sequences as in the proof of lemma 3.6 it is easy to show that $h^{0}\left(S^{\prime}, \mathcal{O}_{S^{\prime}}\left(2 C^{\prime}\right)\right)=3$ and that $h^{0}\left(S^{\prime}, \mathcal{O}_{S^{\prime}}\left(C^{\prime}-E^{\prime}\right)\right)=1$. Thus $|M| \subset\left|3 C^{\prime}\right|$ and $\left|3 C^{\prime}\right|$ is not composed with $\left|C^{\prime}\right|$. From the above considerations it follows that the sequence:

$$
0 \rightarrow H^{0}\left(S^{\prime}, \mathcal{O}_{S^{\prime}}\left(2 C^{\prime}\right)\right) \rightarrow H^{0}\left(S^{\prime}, \mathcal{O}_{S^{\prime}}\left(3 C^{\prime}\right)\right) \rightarrow H^{0}\left(C^{\prime}, \mathcal{O}_{C^{\prime}}\left(3 P_{2}\right)\right) \rightarrow 0
$$

is exact. This amounts to saying that $h^{0}\left(S^{\prime}, \mathcal{O}_{S^{\prime}}\left(3 C^{\prime}\right)\right)=5$ and $P_{2}$ is not a base point of $\left|3 C^{\prime}\right|$. Since $P_{2}$ is the only base point of $\left|C^{\prime}\right|$ by lemma 4.6, it follows that $\left|3 C^{\prime}\right|$ is free. $\diamond$ 
Lemma 4.9 If $S$ is a surface as in assumption 4.1 and $S^{\prime}, C^{\prime}$ are as in notation 4. T, then the linear system $\left|3 C^{\prime}\right|$ defines a morphism $f: S \rightarrow \mathbf{P}^{4}$ of degree 3 onto the normal rational cubic cone $C_{3}$. The curves of $\left|C^{\prime}\right|$ are the pull-backs via $f$ of the rulings of $C_{3}$ and the point $P_{2}$ (see lemma 4.0.) is mapped to the vertex.

Proof: Lemma 4.8 and its proof show that $f$ maps a general curve of $\left|C^{\prime}\right|$ 3-to-1 onto a line in $\mathbf{P}^{4}$ contaning the point $f\left(P_{2}\right)$. So the image of $f$ is a cone with vertex $f\left(P_{2}\right)$. Since $\left(3 C^{\prime}\right)^{2}=9$, the image of $f$ has degree 3 , and so it is the normal rational cubic cone.

Proof of Theorem 4.3: The theorem follows directly from lemmas 4.4, . 4.9. $\diamond$

We close this section by establishing some results on linear systems on $S^{\prime}$ that that will be needed for the contruction of examples of surfaces as in assumption 4.1 with canonical map of degree 3 .

Lemma 4.10 Let $S$ be a surface as in assumption 4.1 such that $\operatorname{deg} \phi_{K}=3$, and let $S^{\prime}, C^{\prime}, E^{\prime}$ be as in notation 4.7 ; then:

i) $h^{0}\left(S^{\prime}, \mathcal{O}_{S^{\prime}}\left(4 C^{\prime}\right)\right)=7$, the point $P_{2}$ (see lemma 4.0) is the only base point of $\left|4 C^{\prime}\right|$ and it is a simple one;

ii) $h^{0}\left(S^{\prime}, \mathcal{O}_{S^{\prime}}\left(5 C^{\prime}\right)\right)=10$ and $\left|5 C^{\prime}\right|$ is base point free;

iii) if $n \geq 6$, then $h^{0}\left(S^{\prime}, \mathcal{O}_{S^{\prime}}\left(n C^{\prime}\right)\right)=11+\frac{n(n-5)}{2}$ and $\left|n C^{\prime}\right|$ is base point free for $n \neq 7$.

Proof: Restricting $\mathcal{O}_{S^{\prime}}\left(4 C^{\prime}\right)$ to a general $C^{\prime}$ and taking global sections one obtains the sequence:

$$
0 \rightarrow H^{0}\left(S^{\prime}, \mathcal{O}_{S^{\prime}}\left(3 C^{\prime}\right)\right) \rightarrow H^{0}\left(S^{\prime}, \mathcal{O}_{S^{\prime}}\left(4 C^{\prime}\right)\right) \rightarrow H^{0}\left(C^{\prime}, \mathcal{O}_{C^{\prime}}\left(4 P_{2}\right)\right) \rightarrow 0 .
$$

By lemma 4.8, the system $\left|3 P_{2}\right|$ on $C$ is free of dimension 2 and it is equal to the restriction of $\left|3 C^{\prime}\right|$ to $C^{\prime}$. Using Riemann-Roch on $C^{\prime}$ and the fact that $C^{\prime}$ is not hyperelliptic, one shows easily that $\left|4 P_{2}\right|=P_{2}+\left|3 P_{2}\right|$. So sequence 4.2 is right exact and claim i) follows now easily from lemma 4.8 .

As we have already remarked in the proof of lemma 4.8, there exists a unique effective divisor $C_{0} \equiv C^{\prime}-E^{\prime}$. By lemma 4.6, the canonical map $\phi_{K}^{\prime}$ of $S^{\prime}$ is induced by the system $|M|=\left|2 C^{\prime}+E^{\prime}\right|$ that has $P_{1}$ as its only base point. Since $M C_{0}=E^{\prime} C_{0}=2$ and $p_{a}\left(C_{0}\right)=3$, it follows that $P_{1} \notin C_{0}$ and $\phi_{K}^{\prime}$ maps $C_{0} 2-$ to-1 onto a ruling of the quadric cone in $\mathbf{P}^{3}$. One has $C^{\prime} C_{0}=0$ and so the restriction of $\mathcal{O}_{S^{\prime}}\left(C^{\prime}\right)$ to $C_{0}$ is trivial. The sequence 
$0 \rightarrow \mathcal{O}_{S^{\prime}}\left(4 C^{\prime}+E^{\prime}\right) \rightarrow \mathcal{O}_{S^{\prime}}\left(5 C^{\prime}\right) \rightarrow \mathcal{O}_{C_{0}} \rightarrow 0$ induces on cohomology the exact sequence:

$$
0 \rightarrow H^{0}\left(S^{\prime}, \mathcal{O}_{S^{\prime}}\left(4 C^{\prime}+E\right)\right) \rightarrow H^{0}\left(S^{\prime}, \mathcal{O}_{S^{\prime}}\left(5 C^{\prime}\right)\right) \rightarrow H^{0}\left(C_{0}, \mathcal{O}_{C_{0}}\right) .
$$

Since the image of $H^{0}\left(S^{\prime}, \mathcal{O}_{S^{\prime}}\left(5 C^{\prime}\right)\right) \rightarrow H^{0}\left(C_{0}, \mathcal{O}_{C_{0}}\right)$ is nonzero, we have $h^{0}\left(S^{\prime}, \mathcal{O}_{S^{\prime}}\left(5 C^{\prime}\right)\right) \geq h^{0}\left(S^{\prime}, \mathcal{O}_{S^{\prime}}\left(4 C^{\prime}+E\right)\right)+1 ;$ to compute $h^{0}\left(S^{\prime}, \mathcal{O}_{S^{\prime}}\left(4 C^{\prime}+E^{\prime}\right)\right)$ we restrict again to $C_{0}$ and consider the sequence on global sections:

$0 \rightarrow H^{0}\left(S^{\prime}, \mathcal{O}_{S^{\prime}}\left(3 C^{\prime}+2 E^{\prime}\right)\right) \rightarrow H^{0}\left(S^{\prime}, \mathcal{O}_{S^{\prime}}\left(4 C^{\prime}+E^{\prime}\right)\right) \rightarrow H^{0}\left(C_{0}, \mathcal{O}_{C_{0}}\left(E^{\prime}\right)\right) \rightarrow 0$.

By the above considerations, $\left|\mathcal{O}_{C_{0}}\left(E^{\prime}\right)\right|$ is the restriction of $|M| \subset\left|4 C^{\prime}+E^{\prime}\right|$ to $C_{0}$ and is a $g_{2}^{1}$. So the sequence is exact and $h^{0}\left(S^{\prime}, \mathcal{O}_{S^{\prime}}\left(4 C^{\prime}+E^{\prime}\right)\right)=$ $h^{0}\left(S^{\prime}, \mathcal{O}_{S^{\prime}}\left(3 C^{\prime}+2 E^{\prime}\right)\right)+2$.

The last step is now the computation of $h^{0}\left(S^{\prime}, \mathcal{O}_{S^{\prime}}\left(3 C^{\prime}+2 E^{\prime}\right)\right)$ : the divisor $3 C=K_{S}+C$ on the surface $S$ is the adjoint of a nef and big divisor, so by Kawamata-Viehweg vanishing $h^{0}\left(S, \mathcal{O}_{S}(3 C)\right)=\chi\left(\mathcal{O}_{S}(3 C)\right)=8$. By the regularity of $S,|3 C|$ restricts to the complete system $\left|K_{C}\right|$ on a generic $C$ and thus $P$ is not a base point of $|3 C|$. It follows $h^{0}\left(S^{\prime}, \mathcal{O}_{S^{\prime}}\left(3 C^{\prime}+2 E\right)\right)=7$, and, finally, $h^{0}\left(S^{\prime}, \mathcal{O}_{S^{\prime}}\left(5 C^{\prime}\right)\right) \geq 10$. Now, taking into account the dimensions of the vector spaces involved, one sees that the sequence:

$$
0 \rightarrow H^{0}\left(S^{\prime}, \mathcal{O}_{S^{\prime}}\left(4 C^{\prime}\right)\right) \rightarrow H^{0}\left(S^{\prime}, \mathcal{O}_{S^{\prime}}\left(5 C^{\prime}\right)\right) \rightarrow H^{0}\left(C^{\prime}, \mathcal{O}_{C^{\prime}}\left(5 P_{2}\right)\right) \rightarrow 0
$$

is exact and therefore $\left|5 C^{\prime}\right|$ is base point free of dimension 9 .

If $n \geq 5$, then $n C^{\prime}=K_{S^{\prime}}+(n-2) C^{\prime}-3 E=K_{S^{\prime}}+(n-5) C^{\prime}+3 C_{0}$ is the adjoint of an effective divisor, and thus $h^{2}\left(S^{\prime}, \mathcal{O}_{S^{\prime}}\left(n C^{\prime}\right)\right)=0$ and $\chi\left(\mathcal{O}_{S^{\prime}}\left(n C^{\prime}\right)\right)=h^{0}\left(S^{\prime}, \mathcal{O}_{S^{\prime}}\left(n C^{\prime}\right)\right)-h^{1}\left(S^{\prime}, \mathcal{O}_{S^{\prime}}\left(n C^{\prime}\right)\right)=5+\frac{n(n-5)}{2}$. By the regularity of $S^{\prime}$, the system $\left|K_{S^{\prime}}+C^{\prime}\right|$ restricts to the complete canonical system $\left|6 P_{2}\right|$ on every curve $C^{\prime}$. One has $6 C^{\prime}=K_{S^{\prime}}+C^{\prime}+3 C_{0}$ and so the restriction map $H^{0}\left(S^{\prime}, \mathcal{O}_{S^{\prime}}\left(6 C^{\prime}\right)\right) \rightarrow H^{0}\left(C^{\prime}, \mathcal{O}_{C^{\prime}}\left(6 P_{2}\right)\right)$ is surjective, implying $h^{0}\left(S^{\prime}, \mathcal{O}_{S^{\prime}}\left(6 C^{\prime}\right)\right)=h^{0}\left(S^{\prime}, \mathcal{O}_{S^{\prime}}\left(5 C^{\prime}\right)\right)+h^{0}\left(C^{\prime}, \mathcal{O}_{C^{\prime}}\left(6 P_{2}\right)\right)=14$ and, as a consequence, $h^{1}\left(S^{\prime}, \mathcal{O}_{S^{\prime}}\left(6 C^{\prime}\right)\right)=6$. Since $h^{1}\left(C^{\prime}, \mathcal{O}_{C^{\prime}}\left(n P_{2}\right)\right)=0$ for $n \geq 7$, one has a surjection $H^{1}\left(S^{\prime}, \mathcal{O}_{S^{\prime}}\left((n-1) C^{\prime}\right)\right) \rightarrow H^{1}\left(S^{\prime}, \mathcal{O}_{S^{\prime}}\left(\left(n C^{\prime}\right)\right)\right.$ for $n \geq 7$. So for $n \geq 6$ one has $h^{1}\left(S^{\prime}, \mathcal{O}_{S^{\prime}}\left(\left(n C^{\prime}\right)\right) \leq 6\right.$ and $h^{0}\left(S^{\prime}, \mathcal{O}_{S^{\prime}}\left(\left(n C^{\prime}\right)\right) \geq 11+\frac{n(n-5)}{2}\right.$, with equality holding for $n=6$. In order to show that equality actually holds for $n \geq 7$, it is enough to prove that the restriction map $H^{0}\left(S^{\prime}, \mathcal{O}_{S^{\prime}}\left(n C^{\prime}\right)\right) \rightarrow$ $H^{0}\left(C^{\prime}, \mathcal{O}_{C^{\prime}}\left(n P_{2}\right)\right)$ is surjective for $n \geq 7$. Using the above discussion, the Riemann-Roch theorem on $C^{\prime}$ and the base point free pencil trick, it is not difficult to prove that the graded ring $\oplus_{n \geq 0} H^{0}\left(C^{\prime}, \mathcal{O}_{C^{\prime}}\left(n P_{2}\right)\right)$ is generated 
by elements of degree $\leq 5$. Since we already know that the restriction map $H^{0}\left(S^{\prime}, \mathcal{O}_{S^{\prime}}\left(n C^{\prime}\right)\right) \rightarrow H^{0}\left(C^{\prime}, \mathcal{O}_{C^{\prime}}\left(n P_{2}\right)\right)$ is surjective for $n \leq 5$, it follows that it is indeed surjective for all $n$. $\diamond$

\section{$5 \quad$ Triple covers of $\mathbf{F}_{3}$}

In the previous sections we have shown that all surfaces satisfying assumption 3.1 and all surfaces satisfying assumption 4.1 and having canonical map of degree 3 are generically finite triple covers of the rational cubic cone $C_{3}$ in $\mathbf{P}^{4}$. In this section we describe these triple covers more precisely, in order to be able to show the existence of the surfaces we are studying.

Triple covers have been studied in [Mi] and [Pa] under the assumption that the map be flat. So we wish to reduce ourselves to the case of a flat map. We state first some general results on coverings that will be useful later:

Lemma 5.1 Let $X, Y$ be surfaces, with $X$ normal and $Y$ smooth and let $f: X \rightarrow Y$ be a quasi-finite projective map: then $f$ is flat.

Proof: The surface $X$, being normal, is Cohen-Macaulay by Theorem 8.22 A of [Ha]; moreover the map $f$ is finite by Exercise 11.2, chapter III of [Ha]. So we may apply Corollary 18.17 of [Ei] to conclude that $X$ is flat over $Y$. $\diamond$

Lemma 5.2 Let $Y$ be a smooth variety and let $\pi: X \rightarrow Y$ be a finite flat map of degree 3 with branch locus $D$, and let $Q$ be a (not necessarily closed) point of $Y$ :

i) if $\pi$ is simply ramified over $Q$, then $Q \in D$ and $X$ is smooth over $Q$ iff $D$ is smooth at $Q$;

ii) if $\pi$ is totally ramified over $Q$, then $D$ is singular at $Q$ and $X$ is smooth over $Q$ iff $D$ has a double point at $Q$.

Proof: This is just a more synthetic formulation of the analysis of section 5 of Mi]. $\diamond$

Lemma 5.3 Let $X, Y$ be irreducible varieties, with $Y$ normal, and let $f$ : $X \rightarrow Y$ be a finite flat map. Then $X$ is normal iff it is nonsingular in codimension 1. 
Proof: It is well known (see [Ha], Thm. 8.22A) that if $X$ is normal, then it is nonsingular in codimension 1 .

Assume now that $X$ is not normal. Then there exists an affine open subset $U=\operatorname{spec} B$ of $X$ that is not normal. Since $f$ is finite (and thus affine), we may assume that $U=f^{-1} V$, where $V=\operatorname{spec} A$ is an affine open subset of $Y$. By the flatness of $f$, possibly after shrinking $V$, there exist $z_{1} \ldots z_{k} \in B$ such that $B=A \oplus A z_{1} \oplus \cdots A z_{k}$. Notice that, if $F$ is the fraction field of $A$, then the fraction field of $B$ is $G=B \otimes_{A} F$. Let $x \in G$ be integral over $B$, but not an element of $B$; then $x=a_{0}+a_{1} z_{1}+\cdots a_{k} z_{k}$ with at least one of the $a_{i}$ 's, say $a_{j}$, not in $A$. Then the locus where $a_{j}$ is not regular is a divisor in $V, Y$ being normal, and thus the set of non-normal points of $X$ has codimension 1. But this contradicts the assumption that $X$ be nonsingular in codimension 1 . $\diamond$

Corollary 5.4 Let $Y$ be a smooth surface and $\pi: X \rightarrow Y$ a finite flat map of degree 3 ; assume that $X$ is not normal and denote by $\tilde{X}$ its normalization: then the map $\tilde{X} \rightarrow Y$ is flat and finite, with branch locus strictly contained in the branch locus of $X \rightarrow Y$.

Proof: The map $\tilde{X} \rightarrow Y$ is flat and finite, by lemma 5.1. Denote by $D$ the branch locus of $X \rightarrow Y$ and by $\tilde{D}$ the branch locus of $\tilde{X} \rightarrow Y$. By the flatness of $\pi$ and $f, D$ and $\tilde{D}$ are divisors and $\tilde{D} \leq D$. By lemma 5.3, if $X$ is not normal then it is singular in codimension 1 and thus, by lemma 5.2, there is an irreducible component $C$ of $D$ such that either $X$ is not totally ramified over $C$ and $C$ appears in $D$ with multiplicity $\geq 2$ or $X$ is totally ramified over $C$ and $C$ appears in $D$ with multiplicity $\geq 3$. In either case $C$ appears in $\tilde{D}$ with multiplicity strictly smaller than in $D$. $\diamond$

Notation 5.5 We denote by $\mathbf{F}_{3}$ the ruled surface $\operatorname{Proj}\left(\mathcal{O}_{\mathbf{P}^{1}} \oplus \mathcal{O}_{\mathbf{P}^{1}}(3)\right)$, by $\sigma_{0}$ a tautological section, by $\sigma_{\infty}$ the infinity section and by $R$ the class of a ruling of $\mathbf{F}_{3}$. So we have $\sigma_{0} \equiv \sigma_{\infty}+3 R, \sigma_{0}^{2}=3, \sigma_{\infty}^{2}=-3, R^{2}=0$. Recall that the linear system $\left|\sigma_{0}\right|$ defines a morphism $\psi: \mathbf{F}_{3} \rightarrow \mathbf{P}^{4}$ that maps $\mathbf{F}_{3}$ birationally onto the cubic cone $C_{3}$ and contracts $\sigma_{\infty}$ to the vertex $v$ of $C_{3}$.

Proposition 5.6 Let $S$ be as in assumption 3.1, let $C_{3}$ be the rational normal cubic cone in $\mathbf{P}^{4}$, let $f: S \rightarrow C_{3}$ be the map induced by the linear system $|3 C|$ (see lemma 3.11), let $\epsilon: \hat{S} \rightarrow S$ be the blow-up of $S$ at the base 
point $P$ of $|C|$ (see lemma 3.5); then there is a commutative diagram:

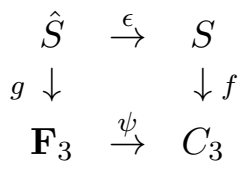

where $g: \hat{S} \rightarrow \mathbf{F}_{3}$ is a generically finite morphism of degree 3 . The map $g$ is totally ramified and finite over $\sigma_{\infty}$.

Before proving the proposition we fix some notation:

Notation 5.7 We denote by $E$ the exceptional curve of $\epsilon: \hat{S} \rightarrow S$; we still denote by $|C|$ the pull-back to $\hat{S}$ of the system $|C|$ on $\hat{S}$, and by $|F|$ the strict transform of $|C|$, so that $|F|$ defines a fibration $\hat{S} \rightarrow \mathbf{P}^{1}$.

Proof of prop. 5.6: In principle, $g$ is a rational map of degree 3; in order to show that $g$ is a morphism we are going to prove that the pull-back on $\hat{S}$ of the very ample linear system $\left|R+\sigma_{0}\right|$ is base point free. By lemma 3.11, one has: $g^{*} R=F=C-E$ and $g^{*} \sigma_{0}=3 C$. By lemma 3.14, the system $|4 C-E|=|3 C+F|$ has dimension $6=\operatorname{dim}\left|R+\sigma_{0}\right|$, and therefore it is equal to $g^{*}\left|R+\sigma_{0}\right|$. The system $|F|$ is free, since it is irreducible and $F^{2}=0$, and the system $|3 C|$ is free by lemma 3.11 , so that $|3 C+F|$ is also free and $g$ is a morphism. The inverse image of $\sigma_{\infty}$ contains the divisor $3 E$, since $\left.3 C\right|_{C}=3 P$ : since $\sigma_{\infty} \equiv \sigma_{0}-3 R$, then $g^{*} \sigma_{\infty} \equiv g^{*}\left(\sigma_{0}-3 R\right) \equiv 3 C-3 R \equiv 3 E$, one gets immediately $g^{*} \sigma_{\infty}=3 E$ and thus $g$ is totally ramified over $\sigma_{\infty}$. $\diamond$

Notation-Remark 5.8 We denote by $\hat{S} \stackrel{h}{\rightarrow} X \stackrel{\pi}{\rightarrow} \mathbf{F}_{3}$ the Stein factorization of $g: \hat{S} \rightarrow \mathbf{F}_{3}$ (see lemma 5.0), so that $X$ is a normal variety, $h$ is a morphism with connected fibres and $\pi: X \rightarrow \mathbf{F}_{3}$ is a finite morphism of degree 3. Notice that by lemma 5.1, $\pi$ is flat. So the theory of [M] and [Pa] applies and $\pi_{*} \mathcal{O}_{X}=\mathcal{O}_{\mathbf{F}_{3}} \oplus \mathcal{E}$, where $\mathcal{E}$ is a locally free sheaf of rank 2 , the so-called trace-zero module of $\pi$.

We determine $\mathcal{E}$ in the next proposition.

Proposition 5.9 Let $S$ be a surface as in assumption 3.1 and let $\pi: X \rightarrow$ $\mathbf{F}_{3}$ and $\mathcal{E}$ be as in notation-remark 5.8. Then

i) if $K_{S}^{2}=7,8,9$ or if $K_{S}^{2}=6$ and $P \in Z$ (see lemma 3.7), then $\mathcal{E}=\mathcal{O}_{\mathbf{F}_{3}}\left(-2 \sigma_{\infty}-4 R\right) \oplus \mathcal{O}_{\mathbf{F}_{3}}\left(-3 \sigma_{\infty}-8 R\right)$

ii) if $K_{S}^{2}=6$ and $P \notin Z$, then $\mathcal{E}=\mathcal{O}_{\mathbf{F}_{3}}\left(-2 \sigma_{\infty}-5 R\right) \oplus \mathcal{O}_{\mathbf{F}_{3}}\left(-3 \sigma_{\infty}-7 R\right)$. 
Proof: Case i): (For $K_{S}^{2}=9$ this is the same as [K], Thm. 2.3). Notice that, by lemma 3.11, $g^{*} R=F$ and, by prop. 5.6, $g^{*} \sigma_{\infty}=3 E$; so if one lets $L=\mathcal{O}_{\mathbf{F}_{3}}\left(2 \sigma_{\infty}+4 R\right)$ and $M=\mathcal{O}_{\mathbf{F}_{3}}\left(3 \sigma_{\infty}+8 R\right)$, one has $g^{*} L=\mathcal{O}_{\hat{S}}(4 F+6 E)=\mathcal{O}_{\hat{S}}(4 C+2 E)$ and $g^{*} M=\mathcal{O}_{\hat{S}}(8 F+9 E)=\mathcal{O}_{\hat{S}}(8 C+E)$. Since $\left(g^{*} L\right) E=-2$, the divisor $2 E$ is contained in the fixed part of $\left|g^{*} L\right|$; on the other hand, by lemma 3.14, $|4 C|$ is base point free of dimension 7 . So $\left|g^{*} L\right|=2 E+|4 C|$, and $g^{*}|L| \subset\left|g^{*} L\right|$ is the 6-dimensional subsystem of curves vanishing on $E$ of order $\geq 3$. Fix a generic $z \in H^{0}\left(\hat{S}, g^{*} L\right)$ : then $z$ vanishes on $E$ of order 2 and is not a pull-back from $\mathbf{F}_{3}$. Arguing as above one sees that $\left|g^{*} M\right|=E+|8 C|$, where $|8 C|$ is free, since $|4 C|$ is, while $g^{*}|M|$ consists of divisors vanishing on $E$ of order $\geq 3$. Again we may choose $w \in H^{0}\left(\hat{S}, g^{*} M\right)$ that vanishes on $E$ precisely of order 1 and is not a pull-back from $\mathbf{F}_{3}$. The pair $(z, w)$ defines a birational morphism $\psi: \hat{S} \rightarrow L \oplus M$ such that $g$ factors through $\psi$. If we can show that the image $Y$ of $\psi$ is a normal surface and the map $Y \rightarrow \mathbf{F}_{3}$ is finite, then it will follow that $\psi: \hat{S} \rightarrow Y$ coincides with $h: \hat{S} \rightarrow X$ by the universal property of the Stein factorization. We start by determining the equations defining $Y$ inside the vector bundle $L \oplus M$. We claim that $H^{0}\left(\hat{S}, g^{*} L^{2}\right)$ is the direct sum of the following subspaces:

$$
V_{0}=g^{*} H^{0}\left(\mathbf{F}_{3}, L^{2}\right), \quad V_{1}=w g^{*} H^{0}\left(\mathbf{F}_{3}, L^{2} \otimes M^{-1}\right) . \quad V_{2}=z g^{*} H^{0}\left(\mathbf{F}_{3}, L\right) .
$$

Notice that, since $g^{*} L^{2}=\mathcal{O}_{\hat{S}}(4 E+8 C)$ and $E C=0,\left|g^{*} L^{2}\right|=4 E+|8 C|$. So $h^{0}\left(\hat{S}, g^{*} L^{2}\right)=26$, by lemma 3.14, and it is easy to check that $\operatorname{dim} V_{0}=18$, $\operatorname{dim} V_{1}=1, \operatorname{dim} V_{2}=7$. Thus it is enough to show that for any relation of the form $s_{0}+s_{1}+s_{2}=0$, with $s_{i} \in V_{i}$, one has $s_{0}=s_{1}=s_{2}=0$. This follows at once by remarking that if $s_{i} \neq 0$ then it vanishes on $E$ with order $i \bmod 3$, for $i=0,1,2$. So $z^{2} \in H^{0}\left(\hat{S}, g^{*} L^{2}\right)$ can be written uniquely as $z^{2}=a z+b w+A$, where $a \in g^{*} H^{0}\left(\mathbf{F}_{3}, L\right), b \in g^{*} H^{0}\left(\mathbf{F}_{3}, L^{2} \otimes M^{-1}\right)$ and $A \in g^{*} H^{0}\left(\mathbf{F}_{3}, L^{2}\right)$. Computations of the same kind yield relations of the form $z w=e z+f w-B$ in $H^{0}\left(\hat{S}, g^{*} L \otimes g^{*} M\right)$ and $w^{2}=c z+d w+C$ in $H^{0}\left(\hat{S}, g^{*} M^{2}\right)$, where $e \in g^{*} H^{0}\left(\mathbf{F}_{3}, M\right), f \in H^{0}\left(\mathbf{F}_{3}, L\right), B \in g^{*} H^{0}\left(\mathbf{F}_{3}, L \otimes\right.$ $M), c \in g^{*} H^{0}\left(\mathbf{F}_{3}, M^{2} \otimes L^{-1}\right), d \in g^{*} H^{0}\left(\mathbf{F}_{3}, M\right)$ and $C \in g^{*} H^{0}\left(\mathbf{F}_{3}, M^{2}\right)$. If we replace $z$ by $z-\frac{a+f}{3}$ and $w$ by $w-\frac{d+e}{3}$, the relations take the form:

$$
\left\{\begin{array}{l}
z^{2}=a z+b w+A \\
z w=-d z-a w-B \\
w^{2}=c z+d w+C
\end{array}\right.
$$

We now wish to show that $A=2\left(a^{2}-b d\right), B=a d-b c$ and $C=2\left(d^{2}-a c\right)$, so that the equations 5.2 are of the same form of those given in Thm. 2.7 
of Mi. To do this, one computes $z^{2} w$ both from the first and the second equation and equates the two expressions thus obtained; this yields a relation involving $z^{2}, z w, w^{2}, z, w$, from which the terms of degree 2 in $w, z$ can be eliminated using equations 5.2 (See lemma 2.4 and lemma 2.6 of [Mi]). So one ends up with a relation of the form $s z+t w+r=0$ in $H^{0}\left(\hat{S}, g^{*}\left(L^{2} \otimes M\right)\right.$, where $s \in g^{*} H^{0}\left(\mathbf{F}_{3}, L \otimes M\right), t \in g^{*} H^{0}\left(\mathbf{F}_{3}, L^{2}\right)$ and $r \in g^{*} H^{0}\left(\mathbf{F}_{3}, L^{2} \otimes M\right)$. Again by considering the vanishing orders on $E$ of the three summands, one deduces $s=t=r=0$. This yields $A=2\left(a^{2}-b d\right), B=b c-a d$. The same elimination procedure for $z w^{2}$ finally gives $C=2\left(d^{2}-a c\right)$.

Equations 5.2 define in $L \oplus M$ a flat finite triple cover $Y^{\prime} \rightarrow \mathbf{F}_{3}$ with trace zero module $\mathcal{E}=L^{-1} \oplus M^{-1}$, and $Y$ is contained in $Y^{\prime}$. Let $U \subset \mathbf{F}_{3}$ be the set of regular values of $g:\left.Y\right|_{U}$ is an étale cover of degree 3 and so $\left.Y\right|_{U}=\left.Y^{\prime}\right|_{U}$. This shows that $Y^{\prime}$ is generically reduced; then, by prop. 3.4 of $\mathrm{Pa}$, it is reduced, and therefore $Y^{\prime}=Y$. Next we are going to show that $Y$ is normal. By the universal property of the Stein factorization, there exists a normalization map $\nu: X \rightarrow Y$ such that $\pi$ is the composition of $Y \rightarrow \mathbf{F}_{3}$ with $\nu$. Denote by $D$ the branch locus of $Y \rightarrow \mathbf{F}_{3}$ and by $D^{\prime}$ the branch locus of $\pi: X \rightarrow \mathbf{F}_{3}$ : by cor. 5.4 $D^{\prime} \leq D$ and, moreover, $D=D^{\prime}$ if and only if $Y$ is normal (and in that case $\nu: X \rightarrow Y$ is an isomorphism). By prop. 7 of [Mi], $D$ is linearly equivalent to $2 \sigma_{\infty}+8 \sigma_{0}$. By prop. 5.6 and lemma 5.2, ii), $D^{\prime}$ contains $2 \sigma_{\infty}$. Let $F \in|F|$ be general: by lemma $3.11 \mathrm{~g}$ maps $F$ 3-to-1 onto a ruling $R$ of $\mathbf{F}_{3}$. Since $F$ is smooth, by lemma $5.2 R$ meets $D^{\prime}-2 \sigma_{\infty}$ outside $\sigma_{\infty}$ and by the Hurwitz formula $\left(D^{\prime}-2 \sigma_{\infty}\right) R=8$. So $D^{\prime}-2 \sigma_{\infty}$ is a divisor linearly equivalent to $8 \sigma_{0}+a R$, with $a \geq 0$. We conclude that $D=D^{\prime}$.

Case ii):

One proceeds exactly as in case i), setting in this case $L=2 \sigma_{\infty}+5 R$ and $M=3 \sigma_{\infty}+7 R$. The dimensions of all the linear systems involved have already been computed in lemma 3.14, ii). $\diamond$

From the analysis of the linear system $|3 C|$ carried out in section 3 it follows that, unless $K_{S}^{2}=9$ or $K_{S}^{2}=6$ and $P \notin Z$, the morphism $g: \hat{S} \rightarrow \mathbf{F}_{3}$ is certainly not finite, and thus the surface $X$ is not smooth. It is possible to give a precise description of the curves contracted by $g$, and thus of the singularities of $X$ in each case, however, since we will not need this, we will just give sufficient conditions on the singularities of a cover $\pi: X \rightarrow \mathbf{F}_{3}$ in order that it arises in the Stein factorization of $g: \hat{S} \rightarrow \mathbf{F}_{3}$ as above. In the next section we will give examples of such singular covers, thus showing that the surfaces that we have described do indeed exist and all possible values 
of the invariants occur.

Proposition 5.10 Let $\pi: X \rightarrow \mathbf{F}_{3}$ be a triple cover with trace zero module $\mathcal{E}$, let $D$ be the branch locus of $\pi$, and let $h: \hat{S} \rightarrow X$ be the minimal desingularization of $X$ :

1) if $\mathcal{E}=\mathcal{O}_{\mathbf{F}_{3}}\left(-2 \sigma_{\infty}-4 R\right) \oplus \mathcal{O}_{\mathbf{F}_{3}}\left(-3 \sigma_{\infty}-8 R\right)$ and $X$ is nonsingular over $\sigma_{\infty}$, then the set-theoretic inverse image of $\sigma_{\infty}$ in $\hat{S}$ is an exceptional curve $E$. If we denote by $S$ the surface obtained by blowing down $E$, then the following are true: 3.1;

1.i) if $X$ is smooth, then $K_{S}^{2}=9, p_{g}(S)=5$, and $S$ satisfies assumption

1.ii) if the only singular point of $X$ is a point $x_{0}$ such that $\pi$ is not totally ramified at $x_{0}$ and $y_{0}=\pi\left(x_{0}\right)$ is a $(3,3)$ point of $D$, then $K_{S}^{2}=8, p_{g}(S)=4$ and $S$ satisfies assumption 3.1 ;

1.iii) if the only singular point of $X$ is a point $x_{0}$ such that $\pi$ is not totally ramified at $x_{0}$ and $y_{0}=\pi\left(x_{0}\right)$ is an ordinary quadruple point of $D$, then $K_{S}^{2}=7, p_{g}(S)=4$ and $S$ satisfies assumption 3.1;

1.iv) if $\pi$ is a Galois cover, then $D=2 \sigma_{\infty}+2 D_{0}$; if $D_{0}$ has an ordinary triple point $y_{0}$ and is smooth elsewhere, then $K_{S}^{2}=6, p_{g}(S)=4$ and $S$ satisfies assumption 3.1 with $P \in Z$ (see lemma 3.7);

2) if $\mathcal{E}=\mathcal{O}_{\mathbf{F}_{3}}\left(-2 \sigma_{\infty}-5 R\right) \oplus \mathcal{O}_{\mathbf{F}_{3}}\left(-3 \sigma_{\infty}-7 R\right)$ and $X$ is nonsingular over $\sigma_{\infty}$, then the inverse image of $\sigma_{\infty}$ in $\hat{S}$ is an exceptional curve $E$, and the surface $S$ obtained by blowing down $E$ has invariants $K_{S}^{2}=6, p_{g}(S)=4$ and satisfies assumption 3.1 with $P \notin Z$ (see lemma 3.X).

Proof: As we have already seen in the proof of thm. 5.9, in case 1) $X$ is defined inside the vector bundle $\mathcal{E}^{\vee}$ by equations 5.2 , where

$a \in H^{0}\left(\mathbf{F}_{3}, \mathcal{O}_{\mathbf{F}_{3}}\left(2 \sigma_{\infty}+4 R\right)\right), b \in H^{0}\left(\mathbf{F}_{3}, \mathcal{O}_{\mathbf{F}_{3}}\left(\sigma_{\infty}\right)\right)$,

$c \in H^{0}\left(\mathbf{F}_{3}, \mathcal{O}_{\mathbf{F}_{3}}\left(4 \sigma_{\infty}+12 R\right)\right), d \in H^{0}\left(\mathbf{F}_{3}, \mathcal{O}_{\mathbf{F}_{3}}\left(3 \sigma_{\infty}+8 R\right)\right) ;$

so $a, b, d$ all vanish on $\sigma_{\infty}$, and thus $\pi$ is totally ramified over $\sigma_{\infty}$ by corollary 4.6 of [Mi]. Analogously, in case 2) we have

$a \in H^{0}\left(\mathbf{F}_{3}, \mathcal{O}_{\mathbf{F}_{3}}\left(2 \sigma_{\infty}+5 R\right)\right), b \in H^{0}\left(\mathbf{F}_{3}, \mathcal{O}_{\mathbf{F}_{3}}\left(\sigma_{\infty}+3 R\right)\right)$,

$c \in H^{0}\left(\mathbf{F}_{3}, \mathcal{O}_{\mathbf{F}_{3}}\left(4 \sigma_{\infty}+9 R\right)\right), d \in H^{0}\left(\mathbf{F}_{3}, \mathcal{O}_{\mathbf{F}_{3}}\left(3 \sigma_{\infty}+7 R\right)\right)$

and so $a, c, d$ all vanish on $\sigma_{\infty}$ and $\pi$ is totally ramified over $\sigma_{\infty}$ also in this case. Thus, both in case 1) and in case 2), if $X$ is smooth then $h^{*} \sigma_{\infty}=3 E$, where $E$ is a smooth rational curve satisfying $(3 E)^{2}=3 \sigma_{\infty}^{2}$, i. e., $E^{2}=-1$.

Denote by $\hat{C}$ the pull-back to $\hat{S}$ of the pencil $|R|$ and by $|C|$ the image of $|\hat{C}|$ in $S$ : under our assumptions, the general $\hat{C}$ (and thus also the general $C$ ) is smooth and has genus 3 by the Hurwitz formula, since $D R=10$. The 
restriction of $\pi \circ h$ maps a smooth $\hat{C} 3-1$ onto a smooth rational curve, and it is easy to check that this implies that $\hat{C}$ (and thus also $C$ ) is not hyperelliptic. Moreover the point $P \in S$ to which $E$ is contracted is the only base point of $|C|$ and it is simple. So $C^{2}=1$ and, by the adjunction formula, $K_{S} C=3$.

Next we compute the singularities of $X$ and the invariants of $S$ in the various cases. In case 1.i), $X$ is smooth, and the formulas of section 8 of Pa] yield $p_{g}(X)=5$ and $K_{X}^{2}=8$, and thus $p_{g}(S)=5, K_{S}^{2}=9$.

In order to compute the invariants of $S$ in cases 1.ii), 1.iii) and 1.iv), in which $X$ is singular, we describe $X$ more precisely. Denote by $V$ the open surface $\mathbf{F}_{3} \backslash \sigma_{\infty}: V$ is isomorphic to the total space of the line bundle $\mathcal{O}_{\mathbf{P}^{1}}(3)$ and, if we denote by $p: V \rightarrow \mathbf{P}^{1}$ the projection, then $p_{*} \mathcal{O}_{V}=$ $\oplus_{k \geq 0} \mathcal{O}_{\mathbf{P}^{1}}(-3 k)$ and the group $\operatorname{Pic}(V)=p^{*} \operatorname{Pic}\left(\mathbf{P}^{1}\right)$ is generated by $L=$ $p^{*} \mathcal{O}_{\mathbf{P}^{1}}(1)$. For $y \in V$, one has $b(y) \neq 0$ in equations 5.2; thus one can eliminate $w$ and obtain a relation of the form $z^{3}+r z+s=0$, where $r \in$ $H^{0}\left(V, L^{8}\right), s \in H^{0}\left(V, L^{12}\right)$. Denote again by $L^{4}$ the total space of the line bundle $L^{4}$ on $V$ and by $q: L^{4} \rightarrow V$ the projection map: then $X \backslash \pi^{-1}\left(\sigma_{\infty}\right)$ is isomorphic to the hypersurface $X_{0}=\left\{z^{3}+r z+s=0\right\} \subset L^{4}$, where $z$ represents the tautological section $q^{*} L^{4}$. We denote again by $\pi: X_{0} \rightarrow V$ the restriction of $\pi: X \rightarrow \mathbf{F}_{3}$; notice that $\pi_{*} \mathcal{O}_{X_{0}}=\mathcal{O}_{V} \oplus L^{-4} \oplus L^{-8}$. The surface $S \backslash P$ is the minimal desingularization of $X_{0}$ and we denote by $\eta: S \backslash P \rightarrow X_{0}$ the resolution map. By the adjunction formula, the dualizing sheaf of $X_{0}$ is $\omega_{X_{0}}=\left.\omega_{L^{4}} \otimes q^{*} L^{12}\right|_{X_{0}}=\left.q^{*} L^{3}\right|_{X_{0}}=\pi^{*} L^{3}$; so $\eta^{*} \omega_{X_{0}}=\mathcal{O}_{S \backslash P}(3 C)$ and, using the projection formula twice, $h^{0}\left(X_{0}, \omega_{X_{0}}\right)=h^{0}\left(V, \pi_{*} \pi^{*} L^{3}\right)=h^{0}\left(V, L^{3}\right)=$ $h^{0}\left(\mathbf{P}^{1}, \mathcal{O}_{\mathbf{P}^{1}}(3)\right)+h^{0}\left(\mathbf{P}^{1}, \mathcal{O}_{\mathbf{P}^{1}}\right)=5$.

By assumption, the surface $X_{0}$ is singular only at the point $x_{0}$ such that $\pi\left(x_{0}\right)=y_{0}$. The singularity $\left(X_{0}, x_{0}\right)$ is analytically isomorphic to the following:

case 1.ii): $u^{2}=f(x, y)$, where $f(x, y)=0$ is the equation of a plane curve having a $(3,3)$ point at the origin;

case 1.iii): $u^{2}=f(x, y)$, where $f(x, y)=0$ is the equation of a plane curve having an ordinary quadruple point at the origin;

case 1.iv): $u^{3}=f(x, y)$, where $f(x, y)=0$ is the equation of a plane curve having an ordinary triple point at the origin.

In all three cases we have an elliptic Gorenstein singularity, whose minimal resolution is well known: the exceptional divisor is an elliptic curve $\Gamma$, such that $\Gamma^{2}=-1$ in case $\left.1 . i\right), \Gamma^{2}=-2$ in case $\left.1 . i i\right)$ and $\Gamma^{2}=-3$ in case 1.iv). A section of $\omega_{X_{0}}$ pulls-back to a regular 2 -form on $S \backslash P$ iff it 
vanishes at $x_{0}$, and $\omega_{S \backslash P}=\eta^{*} \omega_{X_{0}}(-\Gamma)$. Since every regular differential form on $S \backslash P$ extends to a global form on $S$, it follows that $\omega_{S}=\mathcal{O}_{S}(3 C-\Gamma)$ and $p_{g}(S)=h^{0}\left(X_{0}, \omega_{X_{0}}\right)-1=4$. So one has $K_{S}^{2}=9+\Gamma^{2}$, namely $K_{S}^{2}=8$ in case 1.i), $K_{S}^{2}=7$ in case 1. ii) and $K_{S}^{2}=6$ in case 1.iv).

In case 2$), X$ is smooth and the formulas of section 8 of [Pa] yield $p_{g}(X)=4, K_{X}^{2}=5$, and thus $p_{g}(S)=4, K_{S}^{2}=6$.

Remark that, since we have $K_{S}^{2}>0$ and $p_{g}(S)>0$ in all cases, the surface $S$ is of general type.

To show that the surface $S$ satisfies assumption 3.1 in all cases, we only need to show that $S$ is minimal. So assume that this is not the case, and let $\Delta$ be a -1 -curve on $S$. Observe that $C$ is a nef divisor on $S$ and that the only irreducible curves that have zero intersection with $C$ are those contracted by the rational map $S \cdots->\mathbf{F}_{3}$. By the above discussion, no rational curve on $S$ is contracted, and so $\Delta C=m>0$. Let $Y$ be the surface obtained from $S$ by contracting $\Delta$ and let $D$ be the image of $C$ in $Y$; one has $D^{2}=C^{2}+m^{2}=1+m^{2}, K_{Y} D=K_{S} C-m=3-m$. So the index theorem yields: $2 K_{Y}^{2} \leq D^{2} K_{Y}^{2} \leq\left(K_{Y} D\right)^{2} \leq 4$, which contradicts $K_{Y}^{2}=K_{S}^{2}+1 \geq 7$.

Finally, by prop. 5.9, i) we have $P \in Z$ in case 1.iv) and $P \notin Z$ in case 2). $\diamond$

Next we repeat the previous analysis for surfaces as in assumption 4.1 with $\operatorname{deg} \phi_{K}=3$.

Notation 5.11 Let $S$ be a surface satisfying assumption 4.1 and such that $\operatorname{deg} \phi_{K}=3$, let $\epsilon^{\prime}: S^{\prime} \rightarrow S, C^{\prime}, E^{\prime}$ as in notation 4.7, let $\epsilon: \hat{S} \rightarrow S^{\prime}$ be the blow-up of $S^{\prime}$ at the point $P_{2}$, (see lemma 4.0 ), let $E$ be the exceptional curve of $\epsilon$, let $C^{\prime}$ denote again the pull-back of $C^{\prime}$ on $\hat{S}$ and let $F=C^{\prime}-E$.

Proposition 5.12 Let $f: S^{\prime} \rightarrow C_{3}$ be the morphism defined in lemma 4.9; using notation 5.11, there is a commutative diagram:

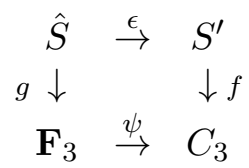

where $g: \hat{S} \rightarrow \mathbf{F}_{3}$ is a finite flat morphism of degree 3 . The map $g$ is totally ramified and finite over $\sigma_{\infty}$.

Proof: The proof is the same as the proof of prop. 5.6. In this case $g^{*}(R+$ $\left.\sigma_{0}\right)=F+3 C^{\prime}$ and, by lemma 4.10, i), the system $\left|F+3 C^{\prime}\right|$ is free of 
dimension $7=\operatorname{dim}\left|R+\sigma_{0}\right|$. So $g^{*}\left|R+\sigma_{0}\right|=\left|F+3 C^{\prime}\right|$ is free and $g$ is a morphism. The last part of the statement is proven as in prop. 5.6. $\diamond$

Notation-Remark 5.13 If $S$ is a surface as in assumption 4.1 and such that $\operatorname{deg} \phi_{K}=3, \hat{S}$ is as in notation $4 . \eta$, we denote by $\hat{S} \stackrel{h}{\rightarrow} X \stackrel{\pi}{\rightarrow} \mathbf{F}_{3}$ the Stein factorization of $g: \hat{S} \rightarrow \mathbf{F}_{3}$ (see prop. 5.12), so that $X$ is a normal variety, $h$ is a morphism with connected fibres and $\pi: X \rightarrow \mathbf{F}_{3}$ is a finite morphism of degree 3. Notice that by lemma 5.1, $\pi$ is flat. So the theory of [M] and [Pd applies and $\pi_{*} \mathcal{O}_{X}=\mathcal{O}_{\mathbf{F}_{3}} \oplus \mathcal{E}$, where $\mathcal{E}$ is a locally free sheaf of rank 2, the so-called trace-zero module of $\pi$.

In the next proposition we determine the trace-zero module $\mathcal{E}$.

Proposition 5.14 Let $S$ be a surface as in assumption 4.1 with $\operatorname{deg} \phi_{K}=3$ and let $\pi: X \rightarrow \mathbf{F}_{3}$ and $\mathcal{E}$ be as in notation-remark 5.19. Then $\mathcal{E}=$ $\mathcal{O}_{\mathbf{F}_{3}}\left(-2 \sigma_{\infty}-5 R\right) \oplus \mathcal{O}_{\mathbf{F}_{3}}\left(-4 \sigma_{\infty}-10 R\right)$.

Proof: The proof is analogous to that of prop. 5.9 and therefore we will only sketch it.

Set $L=\mathcal{O}_{\mathbf{F}_{3}}\left(2 \sigma_{\infty}+5 R\right)$; then by lemma $4.9 g^{*} L=\mathcal{O}_{\hat{S}}(6 E+5 F)=$ $\mathcal{O}_{\hat{S}}\left(5 C^{\prime}+E\right)$ and $\left|g^{*} L\right|=E+\left|5 C^{\prime}\right|$. So lemma 4.10 ii) implies $\operatorname{dim}\left|g^{*} L\right|=9$ and the vanishing order on $E$ of a general element of $H^{0}\left(\hat{S}, g^{*} L\right)$ is precisely 1. A general element $z$ of $H^{0}\left(\hat{S}, g^{*} L\right)$ defines a birational map $\psi: \hat{S} \rightarrow$ $L$ such that $g$ factors through $\psi$. Arguing as in the proof of prop. 5.9 and using lemma 4.10, one can show that $H^{0}\left(\hat{S}, g^{*} L^{3}\right)=g^{*} H^{0}\left(\mathbf{F}_{3}, L\right) \oplus$ $z H^{0}\left(\mathbf{F}_{3}, L^{2}\right) \oplus z^{2} H^{0}\left(\mathbf{F}_{3}, L^{3}\right)$. So there exist $a_{i} \in g^{*} H^{0}\left(\mathbf{F}_{3}, L^{i}\right), i=1,2,3$ such that $z^{3}+a_{1} z^{2}+a_{2} z+a_{3}=0$; up to replacing $z$ by $z+\frac{a_{1}}{3}$, one may assume that $z^{3}+r z+s=0$, where $r \in g^{*} H^{0}\left(\mathbf{F}_{3}, L^{2}\right)$ and $s \in g^{*} H^{0}\left(\mathbf{F}_{3}, L^{3}\right)$. Reasoning again as in the proof of prop. 5.14 one shows that the image of $\psi$ is a normal surface defined inside $L$ by the equation $z^{3}+r z+s=0$ and therefore it is equal to $X$ and $E=L^{-1} \oplus L^{-2}$. $\diamond$

We close this section by stating the analogue of prop. 5.10:

Proposition 5.15 Let $\pi: X \rightarrow \mathbf{F}_{3}$ be a triple cover with trace zero module $\mathcal{E}=\mathcal{O}_{\mathbf{F}_{3}}\left(-2 \sigma_{\infty}-5 R\right) \oplus \mathcal{O}_{\mathbf{F}_{3}}\left(-4 \sigma_{\infty}-10 R\right)$, let $D$ be the branch locus of $\pi$ and let $h: \hat{S} \rightarrow X$ be the minimal desingularization of $X$.

If $X$ is nonsingular over $\sigma_{\infty}$, then the inverse image of $\sigma_{\infty}$ in $\hat{S}$ is an exceptional curve $E$; denote by $S^{\prime}$ the surface obtained by blowing down $E$ and by $P_{2}$ the image point of $E$. If moreover $X$ has only a singular point $x_{0}$ such that $\pi$ is not totally ramified at $x_{0}$ and $y_{0}=\pi\left(x_{0}\right)$ is an ordinary octuple 
point of $D$, then $S^{\prime}$ contains an exceptional curve $E^{\prime}$ such that $P_{2} \in E^{\prime}$, and the surface $S$ obtained from $S^{\prime}$ by contracting $E^{\prime}$ has invariants $K_{S}^{2}=8$, $p_{g}(S)=4$, satisfies assumption 4.1 and the canonical map of $S$ has degree 3 .

Proof: The first statement can be proven exactly as in prop. 5.10 .

The linear system $|\hat{C}|=h^{*} \pi^{*}|R|$ is a base point free pencil of curves of genus 4 , such that the generic element is smooth; moreover, as it was the case in prop. 5.10, the smooth curves of the pencil are not hyperelliptic, since they admit a morphism of degree 3 onto a rational curve. The image $\left|C^{\prime}\right|$ of $|\hat{C}|$ on $S^{\prime}$ has the point $P_{2}$ to which $E$ is contracted as a simple base point, and thus $C^{2}=1, K_{S^{\prime}} C^{\prime}=5$.

Under our assumptions on $\mathcal{E}$, the term $b$ in equations 5.2 is a constant; moreover $b \neq 0$, since otherwise $D_{0}$ would not be reduced by prop. 4.5 of Mi]. So, as in the proof of prop. 5.10, one can eliminate $w$ from equations 5.2 and obtain a relation of the form $z^{3}+r z+s=0$, where $r \in H^{0}\left(\mathbf{F}_{3}, \mathcal{O}_{\mathbf{F}_{3}}\left(4 \sigma_{\infty}+10 R\right)\right)$ and $s \in H^{0}\left(\mathbf{F}_{3}, \mathcal{O}_{\mathbf{F}_{3}}\left(6 \sigma_{\infty}+15 R\right)\right)$. Thus if we denote by $L$ the total space of the line bundle $\mathcal{O}_{\mathbf{F}_{3}}\left(2 \sigma_{\infty}+5 R\right)$ on $\mathbf{F}_{3}$ and by $q: L \rightarrow \mathbf{F}_{3}$ the projection, then $X$ is isomorphic to the hypersurface $\left\{z^{3}+r z+s=0\right\} \subset L$, where $z$ represents the tautological section of $q^{*} L$. By the adjunction formula, the dualizing sheaf of $X$ is $\omega_{X}=\left.\omega_{L} \otimes q^{*} L^{3}\right|_{X}=$ $\left.q^{*}\left(\omega_{\mathbf{F}_{3}} \otimes L^{2}\right)\right|_{X}=\pi^{*} \mathcal{O}_{\mathbf{F}_{3}}\left(2 \sigma_{\infty}+5 R\right)$; using the projection formula, one computes $h^{0}\left(X, \omega_{X}\right)=h^{0}\left(\mathbf{F}_{3}, \mathcal{O}_{\mathbf{F}_{3}}\right)+h^{0}\left(\mathbf{F}_{3}, \mathcal{O}_{\mathbf{F}_{3}}\left(2 \sigma_{\infty}+5 R\right)\right)=10$. Denoting the resolution map by $\eta: \hat{S} \rightarrow X$, one has $\eta^{*} \omega_{X}=\mathcal{O}_{\hat{S}}(6 E+5 \hat{C})$.

The singularity $\left(X, x_{0}\right)$ is analytically isomorphic to the hypersurface singularity $u^{2}+f(x, y)=0$, where $f(x, y)=0$ is the equation of a plane curve with an octuple point at the origin. The minimal resolution of this singularity can be computed by blowing-up the $x, y$-plane at the origin and then taking pull-back and normalization. The exceptional divisor is a smooth hyperelliptic curve $\Gamma$ of genus 3 such that $\Gamma^{2}=-2$ and one has $\omega_{\hat{S}}=\eta^{*} \omega_{X}(-3 \Gamma)$, so that $K_{\hat{S}}^{2}=6$. The condition that the pull-back of a section $\sigma \in H^{0}\left(X, \omega_{X}\right)$ be a regular form on $\hat{S}$ is expressed in local coordinates by requiring $\frac{\partial^{i+j} \sigma}{\partial x^{i} y^{j}}\left(x_{0}\right)=$ for $0 \leq i+j \leq 2$; this amounts to 6 linear conditions and thus $p_{g}(\hat{S}) \geq h^{0}\left(X, \omega_{X}\right)-6=4$. Denote by $R_{0}$ the ruling of $\mathbf{F}_{3}$ that contains $y_{0}$ and write $\eta^{*}\left(\pi^{*} R_{0}\right)=\Gamma+Z$; since $\Gamma+Z \simeq \hat{C}$, one has $\Gamma Z=2, Z^{2}=-2, K_{\hat{S}} Z=0$. Moreover, $Z$ is a finite triple cover of $R_{0}$ totally ramified over the intersection point of $\sigma_{\infty}$ and $R_{0}$, and the total ramification point is a smooth point of $Z$ : it follows easily that $Z$ is irreducible and therefore smooth, since $p_{a}(Z)=0$. Since $Z$ meets $E$ 
transversally, the image $E^{\prime}$ of $Z$ on $S^{\prime}$ is an exceptional curve containing $P_{2}$, and therefore $E^{\prime}$ can be contracted to a smooth point $P$, yielding a surface $S$ with $p_{g}(S)=p_{g}(\hat{S}) \geq 4$ and $K_{S}^{2}=8$. The image of the pencil $\left|C^{\prime}\right|$ is a pencil $|C|$ on $S$, satisfying $C^{2}=2, K_{S} C=4$. The surface $S$ is of general type, since $p_{g}(S)>0$ and $K_{S}^{2}>0$. In order to show that $S$ is minimal, one can argue exactly as in the proof of thm. 5.10. This completes the proof that $S$ satisfies assumption 4.1. Thus, by lemma 4.4, $p_{g}(S)=4$. Again by lemma 4.4, in order to show that $\operatorname{deg} \phi_{K}=3$ it is enough to exclude that $\operatorname{deg} \phi_{K}=4$. So assume by contradiction that $\operatorname{deg} \phi_{K}=4$, and denote by $\Sigma$ the image of $\phi_{K}$ and by $d$ the degree of $\Sigma$ : we have $8=K_{S}^{2} \geq 4 d \geq 4\left(p_{g}(S)-2\right)=8$, and therefore $d=2$ and $\phi_{K}$ is a morphism. Recall that by lemma 4.4 $K_{S}=2 C$. Consider a general $C$ : the system $|3 P|$ is free of dimension 1 by assumption, and the system $|4 P|$ is also free, since it contains the free system $\left|K_{S}\right|_{C}$. It follows that $h^{0}\left(C, \mathcal{O}_{C}(4 P)\right)=3$. On the other hand, we have $K_{C}=6 P$ by the adjunction formula, and therefore the Riemann-Roch theorem gives: $h^{0}\left(C, \mathcal{O}_{C}(4 P)\right)=1+h^{0}\left(C, \mathcal{O}_{C}(2 P)=2\right.$. So we have reached a contradiction. $\diamond$

\section{The examples}

In this section we give explicit examples of surfaces satisfying assumption 3.1 and taking all the possible values of the invariants (see thm. 3.2), and of a surface satisfying assumption 4.1 (in this case the only possibility for the invariants is $K_{S}^{2}=8, p_{g}(S)=4$, by thm. 1.3). We do this by showing that the assumptions in prop. 5.10 and 5.15 can actually be verified. The cases in prop. 6.1, 6.3 and 6.2 are shown to exist by Bertini type arguments.

Proposition 6.1 There exists a surface $S$ satisfying assumption 3.1 with invariants $K_{S}^{2}=9, p_{g}(S)=5$.

Proof: It is enough to show the existence of a smooth triple cover $\pi: X \rightarrow$ $\mathbf{F}_{3}$ as in prop. 5.10, 1.i). As we have already remarked several times, such a cover is determined by the choice of $a \in H^{0}\left(\mathbf{F}_{3}, \mathcal{O}_{\mathbf{F}_{3}}\left(2 \sigma_{\infty}+4 R\right)\right)$, $b \in H^{0}\left(\mathbf{F}_{3}, \mathcal{O}_{\mathbf{F}_{3}}\left(\sigma_{\infty}\right)\right), c \in H^{0}\left(\mathbf{F}_{3}, \mathcal{O}_{\mathbf{F}_{3}}\left(4 \sigma_{\infty}+12 R\right)\right), d \in H^{0}\left(\mathbf{F}_{3}, \mathcal{O}_{\mathbf{F}_{3}}\left(3 \sigma_{\infty}+\right.\right.$ $8 R)$ ). If one takes $a=0, d=0, b \neq 0$ and $c$ such that $c=0$ is a smooth divisor, then it is easy to check using lemma 5.2 and equations 5.2 that the corresponding cover is smooth. Notice that by Bertini's theorem it is possible to find $c$ as required since the linear system $\left|4 \sigma_{0}\right|$ is base point free. $\diamond$ 
Proposition 6.2 There exists a surface $S$ satisfying assumption 3.1 with invariants $K_{S}^{2}=6, p_{g}(S)=4$ and $P \in Z$ (see lemma 3.7).

Proof: By prop. 5.10, 1.iv), we have to show that there exists a Galois triple cover $\pi: X \rightarrow \mathbf{F}_{3}$ branched over $\sigma_{\infty}+D_{0}$, where $D_{0} \in\left|4 \sigma_{0}\right|$ has an ordinary triple point $y_{0} \notin \sigma_{\infty}$ and is smooth elsewhere. This corresponds to taking, in equations 5.2, $a=d=0, b \in H^{0}\left(\mathbf{F}_{3}, \mathcal{O}_{\mathbf{F}_{3}}\left(\sigma_{\infty}\right)\right) \backslash\{0\}$, and $c \in H^{0}\left(\mathbf{F}_{3}, \mathcal{O}_{\mathbf{F}_{3}}\left(4 \sigma_{0}\right)\right)$ such that the divisor $D_{0}=\{c=0\}$ is as above. If the point $y_{0}$ is fixed, then the linear system $W$ of divisors in $\left|4 \sigma_{0}\right|$ having a triple point at $y_{0}$ has no extra base point and the generic element of $W$ has an ordinary triple point at $y_{0}$ : this can be seen easily by considering reducible elements in $W$ given by the sum of three curves of $\left|\sigma_{0}\right|$ passing through $y_{0}$ and of a fourth curve of $\left|\sigma_{0}\right|$ not passing through $y_{0}$. So by Bertini's theorem the generic divisor of $W$ has an ordinary triple point at $y_{0}$ and is smooth elsewhere. $\diamond$

Proposition 6.3 There exists a surface $S$ satisfying assumption 3.1 with invariants $K_{S}^{2}=6, p_{g}(S)=4$ and $P \notin Z$ (see lemma 3.X).

Proof: By prop. 5.10, 2), we have to show that there exists a triple cover $\pi: X \rightarrow \mathbf{F}_{3}$ with $X$ smooth and trace zero module $\mathcal{E}=\mathcal{O}_{\mathbf{F}_{3}}\left(-2 \sigma_{\infty}-5 R\right) \oplus$ $\mathcal{O}_{\mathbf{F}_{3}}\left(-3 \sigma_{\infty}-7 R\right)$. Denote by $\mathbf{P}$ the $\mathbf{P}^{1}$-bundle $\operatorname{Proj}\left(\mathcal{E}^{\vee}\right)$ on $\mathbf{F}_{3}$, by $T$ the tautological hyperplane section on $\mathbf{P}$ and by $p: \mathbf{P} \rightarrow \mathbf{F}_{3}$ the projection. Let $X \subset \mathbf{P}$ be a smooth divisor linearly equivalent to $3 T+p^{*} \operatorname{det} \mathcal{E}$, and assume moreover that $X$ contains no fibre of the map $p$ : then the map $\pi: X \rightarrow \mathbf{F}_{3}$, induced by restricting $p$, is quasi-finite, and therefore flat by lemma 5.1. Thus $\pi: X \rightarrow \mathbf{F}_{3}$ is a triple cover and, by prop. 8.1 of [Mi], its trace zero module is equal to $\mathcal{E}$. So, in order to prove the claim, it is enough to show that the linear system $\left|3 T+p^{*} \operatorname{det} \mathcal{E}\right|$ contains such a divisor $X$.

Recall that $p_{*} \mathcal{O}_{\mathbf{P}}(3 T+\operatorname{det} \mathcal{E})=\mathcal{O}_{\mathbf{F}_{3}}\left(\sigma_{\infty}+3 R\right) \oplus \mathcal{O}_{\mathbf{F}_{3}}\left(2 \sigma_{\infty}+5 R\right) \oplus$ $\mathcal{O}_{\mathbf{F}_{3}}\left(3 \sigma_{\infty}+7 R\right) \oplus \mathcal{O}_{\mathbf{F}_{3}}\left(4 \sigma_{\infty}+9 R\right)$ : since the general section of this vector bundle vanishes nowhere on $\mathbf{F}_{3}$, the general element of $\left|3 T+p^{*} \operatorname{det} \mathcal{E}\right|$ contains no fibre of $p$. Moreover, it is not difficult to check that, as a set, the base locus $B$ of $\left|3 T+p^{*} \operatorname{det} \mathcal{E}\right|$ is the intersection of $p^{-1}\left(\sigma_{\infty}\right)$ with the section of $\mathbf{P}$ corresponding to the quotient map $\mathcal{E}^{\vee} \rightarrow \mathcal{O}_{\mathbf{F}_{3}}\left(2 \sigma_{\infty}+5 R\right)$ and that the general divisor of $\left|3 T+p^{*} \operatorname{det} \mathcal{E}\right|$ is smooth at every point of $B$. It follows from Bertini's theorem that the general divisor in $\left|3 T+p^{*} \operatorname{det} \mathcal{E}\right|$ is smooth. $\diamond$ 
The remaining examples are constructed explicitly, using the symbolic computation program Axiom to show that the assumptions of propositions 5.10 and 5.15 are satisfied. We start by setting some notation. We denote by $\left(t_{0}: t_{1}\right)$ homogeneous coordinates on $\mathbf{P}^{1}$, by $U_{i}$ the open subset $\left\{t_{i} \neq\right.$ $0\} \subset \mathbf{P}^{1}, i=0,1$, by $t=\frac{t_{1}}{t_{0}}$ the affine coordinate on $U_{0}$ and by $s=\frac{t_{0}}{t_{1}}$ the affine coordinate on $U_{1}$. The open surface $V=\mathbf{F}_{3} \backslash \sigma_{\infty}$ is the union of two affine open subsets $V_{i}=U_{i} \times \mathbf{C}, i=0,1$, with coordinates $(t, u)$ and $(s, v)$ respectively, related by $t=\frac{1}{s}, u=s^{3} v$ on $V_{0} \cap V_{1}$.

Proposition 6.4 There exists a surface $S$ satisfying assumption 3.1 with invariants $K_{S}^{2}=8, p_{g}(S)=4$.

Proof: It is enough to show the existence of a triple cover $\pi: X \rightarrow \mathbf{F}_{3}$ as in prop. 5.10, 1.ii). Such a cover is determined by the choice of of $a \in H^{0}\left(\mathbf{F}_{3}, \mathcal{O}_{\mathbf{F}_{3}}\left(2 \sigma_{\infty}+4 R\right)\right), b \in H^{0}\left(\mathbf{F}_{3}, \mathcal{O}_{\mathbf{F}_{3}}\left(\sigma_{\infty}\right)\right), c \in H^{0}\left(\mathbf{F}_{3}, \mathcal{O}_{\mathbf{F}_{3}}\left(4 \sigma_{\infty}+\right.\right.$ $12 R)), d \in H^{0}\left(\mathbf{F}_{3}, \mathcal{O}_{\mathbf{F}_{3}}\left(3 \sigma_{\infty}+8 R\right)\right)$. We take $a=0, b \neq 0$; using lemma 5.2 and lemma 4.5 of [Mi], it is easy to check that if $c$ does not vanish on $\sigma_{\infty}$ then $X$ is nonsingular over $\sigma_{\infty}$. Therefore we only need to study the singularities of $X$ over $V=\mathbf{F}_{3} \backslash \sigma_{\infty}$. We may assume that $b=1$ on $V$ and eliminate $w$ in equations 5.2. Denote by $q: V \rightarrow \mathbf{P}^{1}$ the projection and by $L$ the line bundle $q^{*} \mathcal{O}_{\mathbf{P}^{1}}(4)$ on $V$ : then $\pi^{-1}(V)$ is isomorphic to the hypersurface $\left\{z^{3}+3 d z-c=0\right\} \subset L$, where $z$ represents the tautological section of $L$. On the open set $V_{0}$ we can write: $d=\sum_{i=0}^{2} d_{i} u^{2-i}$, where the $d_{i}$ 's are polynomials in $t$ of degree $2+3 i$, and $c=\sum_{i=0}^{4} c_{i} u^{4-i}$, where the $c_{i}$ 's are polynomials in $t$ of degree $3 i$. The condition that $c$ does not vanish on $\sigma_{\infty}$ corresponds to the condition $c_{0} \neq 0$. Moreover, we assume that the image of the singular point $x_{0}$ is the point $y_{0}=(0,0) \in V_{0}$ (this can always be achieved by means of an automorphism of $\mathbf{F}_{3}$ ). The computations of section 7 show that the required example can be obtained, for instance, by choosing $d=\left(1+t^{2}\right) u^{2}+2 u-t^{8}+t^{7}-t^{6}-1$ and $c=-2 u^{4}-2 u^{3}+6 u-2$. Although we will not give the computations here, we have used Axiom also to determine the coefficients of $d$ and $c$ in such a way that the branch locus $D$ of the cover has a $(3,3)$-point at $y_{0}$. $\diamond$

Proposition 6.5 There exists a surface $S$ satisfying assumption 3.1 with invariants $K_{S}^{2}=7, p_{g}(S)=4$.

Proof: It is enough to show the existence of a triple cover $\pi: X \rightarrow \mathbf{F}_{3}$ as in prop. 5.10, 1.iii). We argue as in the proof prop. 6.4 and, using the same 
notation, we take here: $a=0, b \neq 0, c=-2 u^{4}-20 u^{3}-6 u^{2}+12 u+2 t^{12}-$ $2 t^{11}-2, d=2 u^{2}+4 u+t^{4}-1$. Also in this case, we have used Axiom to determine the coefficients of $d$ and $c$ in such a way that the branch locus $D$ of the cover has an ordinary quadruple point at $y_{0}$. $\diamond$

Proposition 6.6 There exists a surface $S$ satisfying assumption 4.1. As predicted by thm. 4.马, the invariants of $S$ are $K_{S}^{2}=8, p_{g}(S)=4$.

Proof: It is enough to show the existence of a triple cover $\pi: X \rightarrow \mathbf{F}_{3}$ as in prop. 5.15; using a notation consistent with the one in the proof of prop. 5.15, we wish to find $r \in H^{0}\left(\mathbf{F}_{3}, \mathcal{O}_{\mathbf{F}_{3}}\left(4 \sigma_{\infty}+10 R\right)\right.$ and $s \in H^{0}\left(\mathbf{F}_{3}, \mathcal{O}_{\mathbf{F}_{3}}\left(6 \sigma_{\infty}+\right.\right.$ $15 R)$ such that the hypersurface $X=\left\{z^{3}+r z+s=0\right\} \subset \mathcal{O}_{\mathbf{F}_{3}}\left(2 \sigma_{\infty}+5 R\right)$ satisfies the assumptions of prop. 5.15. Notice that $X$ is smooth over $\sigma_{\infty}$ iff $s$ vanishes on $\sigma_{\infty}$ of order 1 . So, as in the previous cases, we only study the restriction of $X$ to $V$ and assume that $y_{0}=(0,0) \in V_{0}$. Using the coordinates of $V_{0}$, we write $r=\sum_{i=0}^{3} r_{i} u^{3-i}$, where $r_{i}$ is a polynomial in $t$ of degree $3 i+1$ and $s=\sum_{i=0}^{5} s_{i} u^{5-i}$, where $s_{i}$ is a polynomial in $t$ of degree $3 i$. By the computations of section 0 , we may take: $r=36 u^{3}-45 u^{2}+18 u-$ $3+3 t^{10}-3 t^{9}+3 t^{8}$ and $s=-27 u^{5}+135 u^{4}-144 u^{3}+72 u^{2}-18 u+2$. Also in this case, we have used Axiom to determine the coefficients of $d$ and $c$ in such a way that the branch locus $D$ of the cover has an ordinary octuple point at $y_{0}$. $\diamond$

\section{Appendix: computations with Axiom}

This section contains the computations with Axiom that are needed in the proofs of propositions 6.4, 6.5 and 6.6. We give a slightly edited version of the Axiom session for prop. 6.6, which shows the existence of the "new" example, and only the input sequences for prop. 6.4 and 6.5, which are very similar to the first one. The notation is consistent with the one defined in the previous section, the only difference is that in the first example the affine coordinate at infinity on $\mathbf{P}^{1}$ is denoted by $y$ instead of $s$.

Prop. 6.6:

We start by checking that the only singular point of $X$ over $U_{0}$ is the point $z=1, u=t=0$

initial (60) $\rightarrow r: P:=36 * u \wedge 3-45 * u \wedge 2+18 * u-3+3 * t \wedge 10-3 * t \wedge 9+3 * t \wedge 8$

$$
36 u^{3}-45 u^{2}+18 u+3 t^{10}-3 t^{9}+3 t^{8}-3
$$


Type: Polynomial Fraction Integer initial (61) $\rightarrow \mathrm{s}: \mathrm{P}:=-27 * \mathrm{u} \wedge 5+135 * \mathrm{u} \wedge 4-144 * \mathrm{u}^{\wedge} 3+72 * \mathrm{u}^{\wedge} 2-18 * \mathrm{u}+2$

(61) $-27 u^{5}+135 u^{4}-144 u^{3}+72 u^{2}-18 u+2$

Type: Polynomial Fraction Integer initial (62) $\rightarrow f: P:=z^{\wedge} 3+r * z+s$

(62) $z^{3}+\left(36 u^{3}-45 u^{2}+18 u+3 t^{10}-3 t^{9}+3 t^{8}-3\right) z-27 u^{5}+135 u 4-$ $144 u^{3}+72 u^{2}-18 u+2$

initial (63) $\rightarrow$ fz:P:=differentiate $(f, z)$

Type: Polynomial Fraction Integer

$$
3 z^{2}+36 u^{3}-45 u^{2}+18 u+3 t^{10}-3 t^{9}+3 t^{8}-3
$$

Type: Polynomial Fraction Integer initial (64) $\rightarrow$ ft:P:=differentiate $(f, t)$

$$
\left(30 t^{9}-27 t^{8}+24 t^{7}\right) z
$$

initial (65) $\rightarrow$ fu:P:=differentiate $(f, u)$

Type: Polynomial Fraction Integer

$$
\left(108 u^{2}-90 u+18\right) z-135 u^{4}+540 u^{5}-432 u^{2}+144 u-18
$$

Type: Polynomial Fraction Integer

initial (66) $\rightarrow$ sing: List $P:=[f, f z, f t, f u]$

(66) $\left[z^{3}+\left(36 u^{3}-45 u^{2}+18 u+3 t^{10}-3 t^{9}+3 t^{8}-3\right) z-27 u^{5}+135 u 4-\right.$ $144 u^{3}+72 u^{2}-18 u+2,3 z^{2}+36 u^{3}-45 u^{2}+18 u+3 t^{10}-3 t^{9}+3 t^{8}-3,\left(30 t^{9}-\right.$ $\left.\left.27 t^{8}+24 t^{7}\right) z,\left(108 u^{2}-90 u+18\right) z-135 u^{4}+540 u^{5}-432 u^{2}+144 u-18\right]$

Type: List Polynomial Fraction Integer

initial (67) $\rightarrow$ solve sing +++ Garbage collection 27 (internal list2*) after 177.89+65.34 seconds At gc end about 5.5Mbytes of $14.0(39.3 \%)$ of heap is in use (67) $\quad[[z=1, u=0, t=0]]$

Type: List List Equation Fraction Polynomial Integer 
Next we compute the discriminant of $f$ with respect to the variable $z$, which is an equation for the branch locus of the cover $\left.X\right|_{U_{0}} \rightarrow U_{0}$. It is easy to check that it has an ordinary octuple point at $t=u=0$.

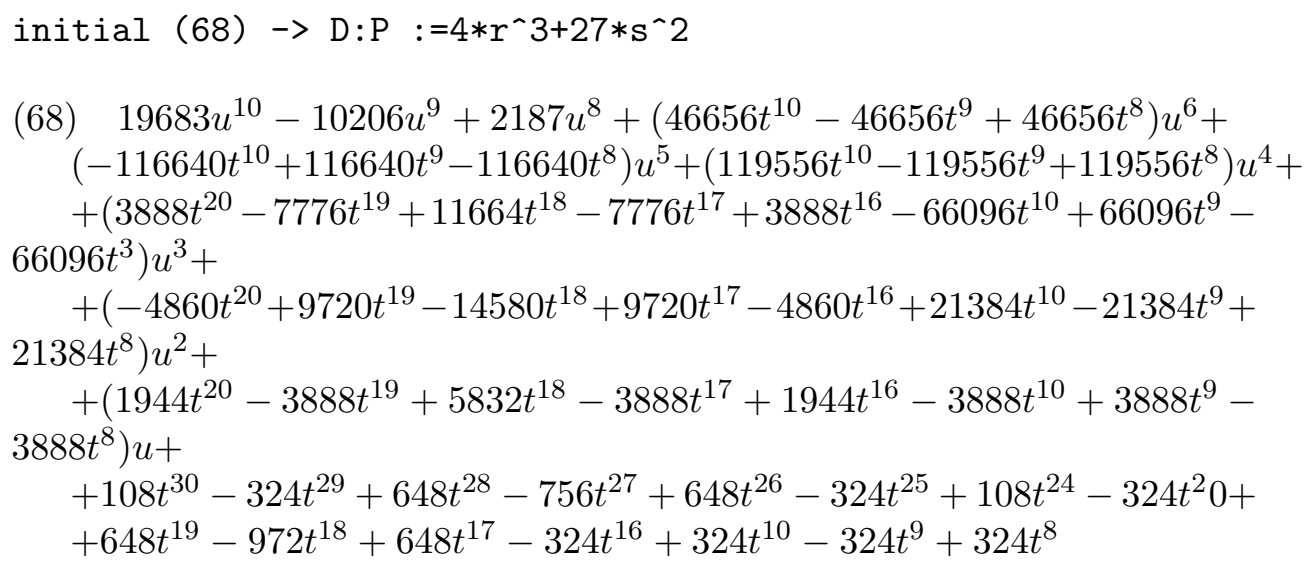

Type: Polynomial Fraction Integer

Finally we show that $X$ is smooth above the line $\{y=0\} \subset U_{1}$, where $y=$ $1 / t$ is the affine coordinate at infinity on $\mathbf{P}^{1}$. We denote by $f_{0}=z^{3}+r_{0} z+s_{0}$ the equation of $X$ over the open set $U_{1}$.

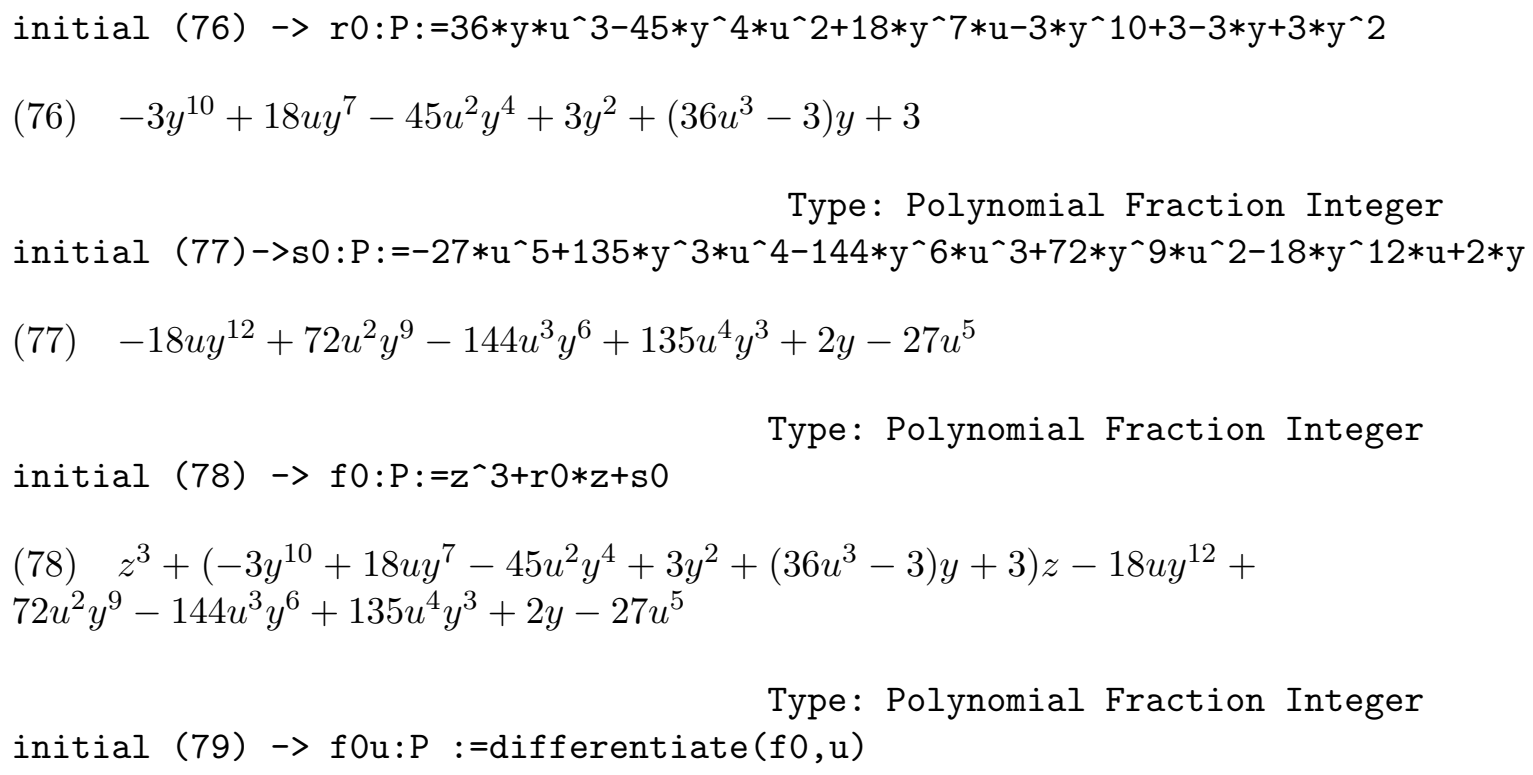$$
-3 y^{10}+18 u y^{7}-45 u^{2} y^{4}+3 y^{2}+\left(36 u^{3}-3\right) y+3
$$

Type: Polynomial Fraction Integer

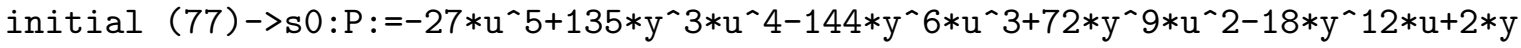$$
-18 u y^{12}+72 u^{2} y^{9}-144 u^{3} y^{6}+135 u^{4} y^{3}+2 y-27 u^{5}
$$

Type: Polynomial Fraction Integer

initial (78) $\rightarrow \mathrm{f} 0: P:=z^{\wedge} 3+r 0 * z+s 0$

(78) $z^{3}+\left(-3 y^{10}+18 u y^{7}-45 u^{2} y^{4}+3 y^{2}+\left(36 u^{3}-3\right) y+3\right) z-18 u y^{12}+$ $72 u^{2} y^{9}-144 u^{3} y^{6}+135 u^{4} y^{3}+2 y-27 u^{5}$

initial (79) $\rightarrow$ fOu:P :=differentiate $(f 0, u)$

Type: Polynomial Fraction Integer 


$$
\left(18 y^{7}-90 u y^{4}+108 u^{2} y\right) z-18 y^{12}+144 u y^{9}-432 u^{2} y^{6}+540 u^{3} y^{3}-135 u^{4}
$$

Type: Polynomial Fraction Integer initial (80) $\rightarrow$ fOy:P :=differentiate $(f 0, y)$

(80) $\left(-30 y^{9}+126 u y^{6}-180 u^{2} y^{3}+6 y+36 u^{3}-3\right) z-216 u y^{11}+648 u^{2} y^{8}+$ $-864 u^{3} y^{5}+405 u^{4} y^{2}+2$

initial (81) $\rightarrow$ f0z:P :=differentiate $(f 0, z)$

Type: Polynomial Fraction Integer

$$
3 z^{2}-3 y^{10}+18 u y^{7}-45 u^{2} y^{4}+3 y^{2}+\left(36 u^{3}-3\right) y+3
$$

Type: Polynomial Fraction Integer initial (82) $\rightarrow$ sing $0:$ List $P:=[f 0, f 0 z, f 0 y, f 0 u, y]$

(82) $\left[z^{3}+\left(-3 y^{10}+18 u y^{7}-45 u^{2} y^{4}+3 y^{2}+\left(36 u^{3}-3\right) y+3\right) z-18 u y^{12}+\right.$ $72 u^{2} y^{9}-144 u^{3} y^{6}+135 u^{4} y^{3}+2 y-27 u^{5}, 3 z^{2}-3 y^{10}+18 u y^{7}-45 u^{2} y^{4}+3 y^{2}+$ $\left(36 u^{3}-3\right) y+3,\left(-30 y^{9}+126 u y^{6}-180 u^{2} y^{3}+6 y+36 u^{3}-3\right) z-216 u y^{11}+$ $648 u^{2} y^{8}+-864 u^{3} y^{5}+405 u^{4} y^{2}+2,\left(18 y^{7}-90 u y^{4}+108 u^{2} y\right) z-18 y^{12}+$ $\left.144 u y^{9}-432 u^{2} y^{6}+540 u^{3} y^{3}-135 u^{4}, y\right]$

initial (83) $\rightarrow$ solve $\%$

(83) $[[]]$

Type: List Polynomial Fraction Integer

Polynomial Integer

Type: List List Equation Fraction

Prop. 6.4:

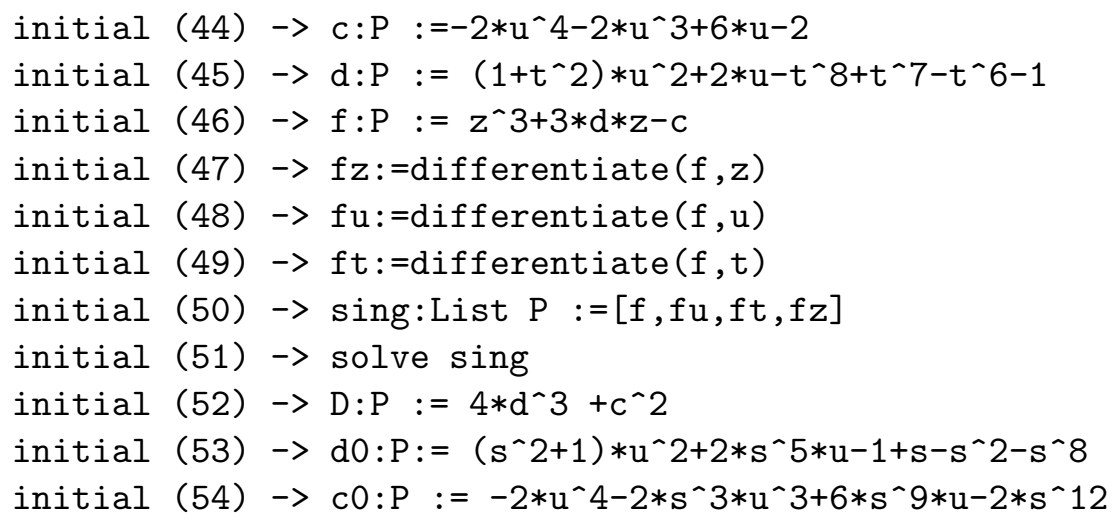




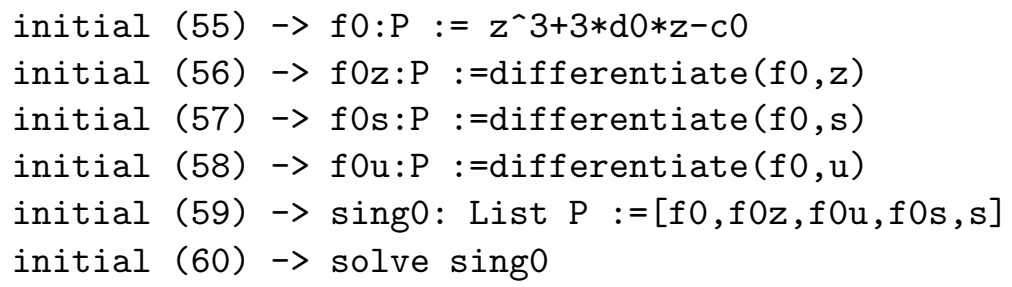

Prop. 6.5:

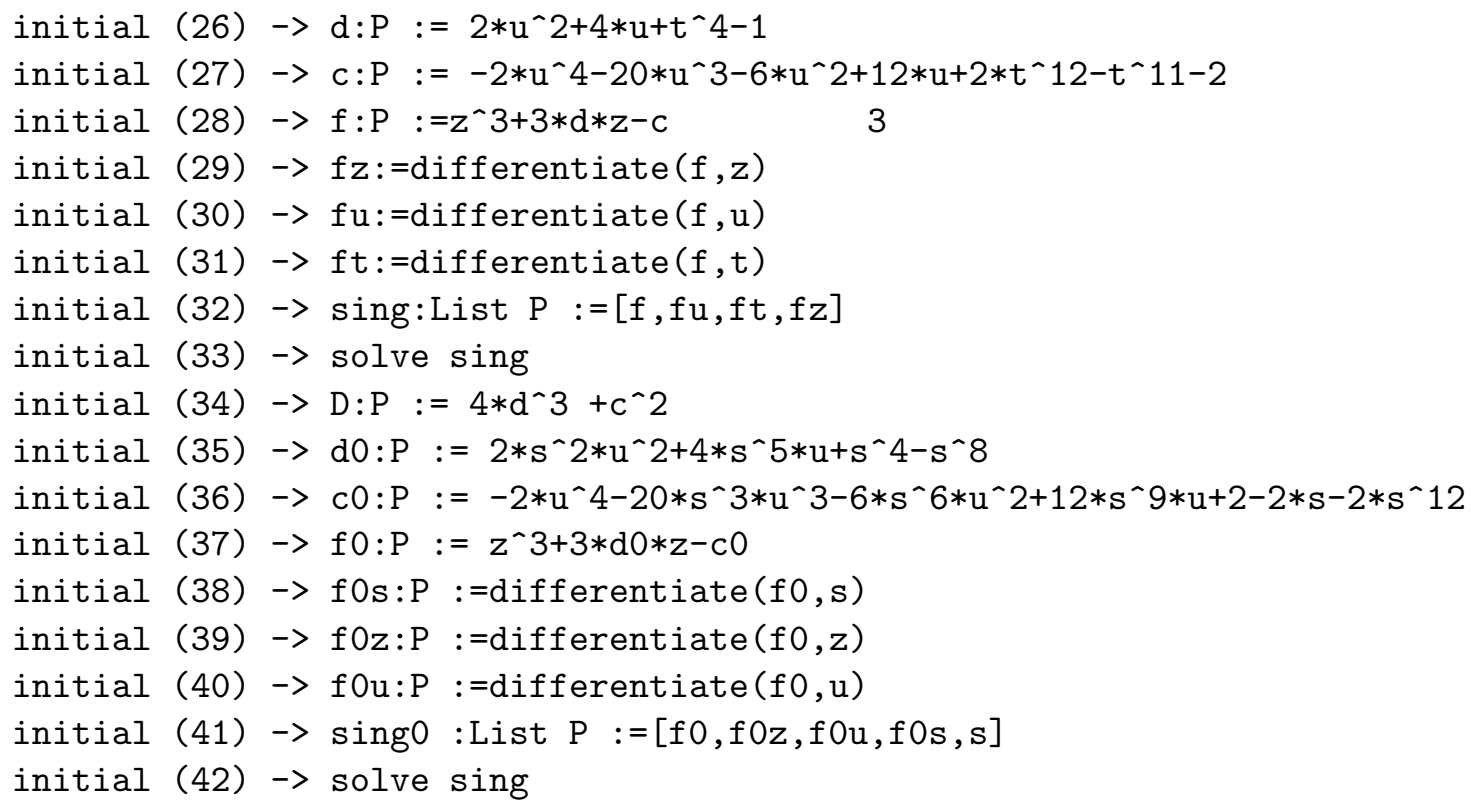

\section{References}

[B] E. Bombieri, Canonical models of surfaces of general type, Publ. Math. IHES, 42 (1973), 171-219.

[CFM] C. Ciliberto, P. Francia, M. Mendes Lopes, Remarks on the bicanonical map for surfaces of general type, Math. Zeit., 224 (1997), 137-166.

[De] O. Debarre, Inégalités numériques pour les surfaces de type general, Bull. Soc. Math. de France, 110 (1982), 319-346

[Ei] D. Eisenbud, Commutative algebra with a view toward algebraic geometry, G.T.M. 150, Springer-Verlag, New York (1995). 
[F] P. Francia, On the base points of the bicanonical system, in Problems in the theory of surfaces and their classification, Symposia Math., Academic Press, 1991, 141-150.

[Ha] R. Hartshorne, Algebraic Geometry, G.T.M. 52, Springer-Verlag, New York (1977).

[Ho1] E. Horikawa, Algebraic surfaces of general type with small $c_{1}^{2}$, I, Ann. Math., 104 (1976), 357-387

[Ho2] E. Horikawa, Algebraic surfaces of general type with small $c_{1}^{2}$, III, Inv. Math., 47 (1978), 209-248

[Ho3] E. Horikawa, Algebraic surfaces of general type with small $c_{1}^{2}$, V, J. Fac. Sci. Univ. Tokyo, Sec. IA, Math. 28 (1981) (3), 745-755

[K] K. Konno, Algebraic surfaces of general type with $c_{1}^{2}=3 p_{g}-6$, Math. Ann., 290 (1991), 77-107.

[Mi] R. Miranda, Triple covers in algebraic geometry, American J. of Math., 107, No 5, (1985), 1123-1158.

[M] M. Mendes Lopes, Adjoint systems on surfaces, Boll.Unione Matematica Italiana, (7) 10-A (1996), 169-179.

[MP] M. Mendes Lopes, R. Pardini, Irregular canonical double surfaces, to appear in Nagoya Math. Jour.

[Pa] R. Pardini, Triple covers in positive characteristic, Arkiv för matematik 27, No 2, (1989), 319-341.

[Pe] U. Persson, On Chern invariants of surfaces of general type, Compos. Math, 43 (1981), 3-58.

[Po] G. Pompilj, Sulle superficie algebriche le cui curve canoniche posseggono una $g_{3}^{1}$., Ist. Lombardo. Sci. Lett. Cl. Sci. Mat. Nat. Rend., (3) 5(74), 1941, 280-286.

[St] F. Starnone, Superficie di tipo generale con curve canoniche trigonali, thesis, Roma, 1997.

[Z] F. Zucconi, Numerical inequalities for surfaces with canonical map composed with a pencil, Indagationes Mathematicae, N.S., 8 (3), 1-18 
Margarida Mendes Lopes

Centro de Algebra

Universidade de Lisboa

Av. Prof. Gama Pinto, 2

1699 Lisboa Codex, PORTUGAL

mmlopes@lmc.fc.ul.pt
Rita Pardini

Dipartimento di Matematica Università di Pisa

Via Buonarroti 2

56127 Pisa, ITALY

pardini@dm.unipi.it 